\title{
Mihail Benado
}

Les ensembles partiellement ordonnés et le théorème de raffinement de Schreier. I

Czechoslovak Mathematical Journal, Vol. 4 (1954), No. 2, 105-129

Persistent URL: http://dml.cz/dmlcz/100103

\section{Terms of use:}

(C) Institute of Mathematics AS CR, 1954

Institute of Mathematics of the Czech Academy of Sciences provides access to digitized documents strictly for personal use. Each copy of any part of this document must contain these Terms of use.

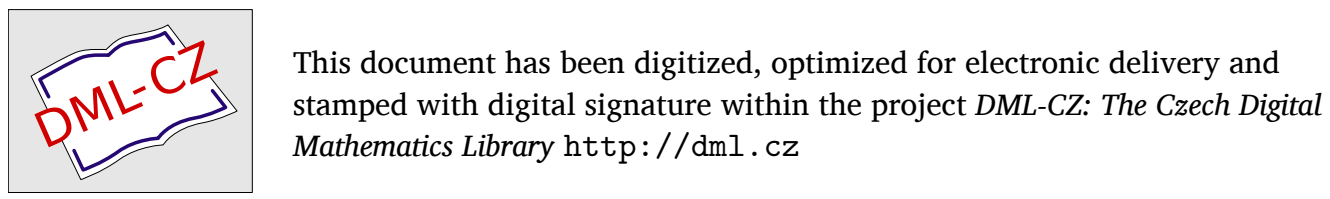




\title{
LES ENSEMBLES PARTIELLEMENT ORDONNÉS ET LE THÉOREMME DE RAFFINEMENT DE SCHREIER, I
}

\author{
MIHAIL BENADO, Bucarest.
}

(Reçu le 6 février 1953.)

\begin{abstract}
Dans ce mémoire il s'agît de l'extension du théorème de Schreier aux ensembles partiellement ordonnés qui satisfont à une certaine condition.

On y donne aussi quelques applications du théorème de Schreier.
\end{abstract}

\section{INTRODUCTION}

1. On doit à Otтo Schreier [1] le théorème suivant qui joue, comme on sait, un rôle fondamental dans la théorie des chânes normales du groupe abstrait (S):

Deux chaînes normales (finies) aux extrémités communes $\mathbb{G} \geqq F$

$$
\left.\begin{array}{l}
\text { (S) } \geqq G_{1} \geqq \cdots \geqq G_{i-1} \geqq G_{i} \geqq \cdots \geqq G_{r-1} \geqq G_{r}=F \\
\text { (S) } \geq H_{1} \geq \ldots \geq H_{j-1} \geq H_{j} \geq \ldots \geq H_{s-1} \geq H_{s}=F
\end{array}\right\}
$$

où $F$ est diviseur normal dans $\mathfrak{G}$, possèdent des raffinements isomorphes.

C'est H. Zassenhaus [2] qui, le premier, trouva les formules explicites des raffinements requis*). A. KuRosch [3], trouva les mêmes formules dans le cas des chaînes normales infinies (,,systèmes normaux" dans la terminologie de Kurosch, 1. c.) munies d'un bon ordre ascendant.

2. Mais, ce fut seulement la théorie des structures qui permît aux algébristes l'axiomatisation du théorème de Schreier. Parmi les nombreuses contributions à ce sujet, je citerai celles de A. Kurosch [4], O. Ore [5, 6], A. Halanay [7], A. I. Ouzkov [8], E. George [9], V. KoŘínek [10], A. H. Lipschitz [13] et D. Barbilian [14]. Parmi ces contributions, celle de Ouzkov est d'une importance particulière, précisément parce que c'est là que pour la première fois ont été démontrées des conditions nécessaires et suffisantes afin que le

*) Il importe de remarquer que la démonstration de Zassenhaus (l. c.) du théorème de Schreier, contient en puissance une certaine généralisation de ce théorème; en effet, si l'on y remplace la supposition , $F$ diviseur normal de $\mathbb{S}^{\prime \prime}$ par la supposition moins exigeante ,$F^{\prime}$ diviseur normal de $G_{r-1}$ et de $H_{s-1}$ " (voir les (I)), le théorème reste vrai. Voir aussi, à ce sujet, les mémoires $[4,7]$. 
théorème de raffinement de Schreier soit vrai dans une structure sous une certaine forme très générale.

3. Reprenant les recherches de Ore [6], d'Ouzkov (l. c.) et tenant compte de la distinction très importante, due à Kořínek (l. c.), entre les deux parties $\mathrm{du}$ théorème de raffinement de Schreier, je suis parvenu tout récemment $[16,17]$ à élargir encore les résultats de ces auteurs par des méthodes se rattachant à la théorie des connexions monotones.

4. Or, il m'est apparu que maints raisonnements aboutissant aux diverses formes du théorème de raffinement de Schreier, ne devaient leur validité qu'aux seules propriétés formelles de certains systèmes de connexions monotones attachées à la structure; ces propriétés ne requièrent, d'ailleurs, que les seuls termes de l'ordre partiel, c'est-à-dire que pour assurer la validité du théorème de Schreier dans un ensemble partiellement ordonné $P$, il n'est point nécessaire (et, en général, il n'est pas même suffisant) que $P$ soit une structure mais, il suffit que certaines conditions de ,,connexité“ y soient vérifiées, à savoir:

Supposition $\boldsymbol{H}$. Nous dirons qu'un ensemble partiellement ordonné $P$ satisfait $\grave{a}$ la supposition $\boldsymbol{H}$, lorsqu'il y existe un élément $\omega_{0}$ tel que pour tout quadrilatère $\left(\Omega, \omega_{0} ; a, b\right)$ on puisse toujours trouver un sousquadrilatère $(M, d ; a, b)$ de façon $q u$ 'on ait les isomorphismes (au sens de l'ordre partiel [19]) $\eta, \tilde{\eta}$ suivants

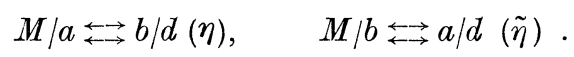

5. Le principal but de la première partie de notre mémoire est précisément de faire voir que cette supposition $\boldsymbol{H}$ est en effet suffisante pour assurer la validité du théorème de raffinement de Schreier dans un ensemble partiellement ordonné (arbitraire).

On y trouve aussi quelques simples applications à la théorie des $W$-structures (M. WARD [18]) et à la théorie de la divisibilité; mais, ce n'est que dans la seconde partie de ce mémoire qu'on pourra surtout se rendre compte de la portée de cette généralisation du théorème de Schreier.

\section{§ 1. DÉFINITIONS ET NOTATIONS GÉNÉRALES}

Nous allons d'abord rappeler les définitions suivantes:

1.1. Ensemble quotient. Soit $P$ un ensemble partiellement ordonné [19], et soient $a, b \in P$ tels que $a \geqq b$, J'appele en sem ble quotient ou simplement. quotient $a / b$, l'ensemble de tous les éléments $x \in P$ tels que $a \geqq x \geqq b$. Si l'on a $a>b$, le quotient est véritable; et tout quotient de la forme $a / a$ sera. dit unitaire. Enfin, je dis qu'un quotient $a / b$ est premier, lorsqu'il est véritable $(a>b)$ et lorsqu'il n'existe aucun élément $x \in P$ tel que $a>x>b$.

1.2. Chaînes. Je dis qu'un sous-ensemble $C$ d'un ensemble partiellement ordonné $P$, est une chaîne (ou ensemble ordonné), lorsque pour tout couple 
$a, b \epsilon C$ on a $a \geqq b$ ou $a \leqq b$. Lorsqu'une chaîne est finie, on peut l'écrire simplement

$$
a_{0} \geqq a_{1} \geqq \ldots \geqq a_{i-1} \geqq a_{i} \geqq \ldots \geqq a_{r-1} \geqq a_{r}, \quad r \geqq 1 .
$$

Les quotients $a_{i-1} / a_{i}, i=1,2, \ldots, r$, sont les quotients ou facteurs de la chaîne; le nombre $r$, en est la longueur (apparente) ${ }^{1}$ ) et les éléments $a_{0}, a_{r}$ en sont les extrémités.

Nous ne considérerons dans ce travail que des chaînes finies de sorte que je dirai toujours ,,chaîne“ tout court, au lieu de ,chaîne finie“.

Je dis qu'un ensemble partiellement ordonné est archimédien ${ }_{1}$ resp. archimédien $_{2}$ s'il satisfait à la condition des chaînes descendantes ${ }^{\text {Ibis }}$ resp. ascendantes ${ }^{\text {1tis }}$; et je dis qu'un ensemble partiellement ordonné est archimédien s'il est à la fois archimédien ${ }_{1}$ et archimédien $_{2}$.

J'appelle raffinement de la chaîne $C$, toute chaîne $C^{\prime}, a_{0}^{\prime} \geqq a_{\mathbf{1}}^{\prime} \geqq \ldots \geqq a_{s}^{\prime}$ telle que $a_{0}=a_{0}^{\prime}, a_{r}=a_{s}^{\prime}$ et telle que tout $a_{i} \epsilon C$ soit un $a_{j}^{\prime} \in C^{\prime}$.

Une chaîne dont tous les quotients sont premiers, sera dite série.

Les définitions que je vais maintenant poser, sont plus spécifiquement liées aux recherches du présent mémoire.

1.3. Quadrilatère de Dedekind. Soit $P$ un ensemble partiellement ordonné; j'appelle quadrilatère de Dedekind ou simplement quadrilatère toute paire de chaînes de longueur apparente égale à 2 , aux extrémités communes

$$
\Omega \geqq a \geqq \omega, \quad \Omega \geqq b \geqq \omega \quad(\Omega, \omega, a, b \in P) .
$$

Les quotients des chaînes $(Q)$ sont les côtes du quadrilatère; ces côtés, sont deux à deux opposés, savoir $\Omega / a$ à $b / \omega$ et $\Omega / b$ à $a / \omega$.

Pour des raisons d'abréviation, je vais utiliser le symbole $(\Omega, \omega ; a, b)$ comme équivalent des relations $(Q)$; $c$ 'est à dire que $(\Omega, \omega ; a, b)$ est le symbole d'un quadrilatère de Dedekind.

Je dis qu'un quadrilatère $(\Omega, \omega ; a, b)$ est à côtés opposés semblables, lorsque l'une des quatre combinaisons suivantes

1. $\Omega>a, b>\omega ; \Omega>b, a>\omega$, 3. $\Omega=a, b=\omega ; \Omega>b, a>\omega$,

2. $\Omega>a, b>\omega ; \Omega=b, a=\omega, \quad 4 . \Omega=a, b=\omega ; \Omega=b, a=\omega$,

est vérifiée.

Ainsi, par exemple, le quadrilatère $(\Omega, \omega ; a, b)$ où l'on a $a$ non $\geqq b$ et $a$ non $\leqq b$, est un quadrilatère à côtés opposés semblables car, on a évidemment $\Omega>a$, $b>\omega, \Omega>b, a>\omega$.

J'appelle sousquadrilatère d'un quadrilatère $(\Omega, \omega ; a, b)$, un quadrilatère $(M, d ; a, b)$ tel que $M \leqq \Omega, d \geqq \omega$; et je dis que $(\Omega, \omega ; a, b)$ est irréductible

1) Lorsque tous les quotients de la chaîne $C$ sont véritables, on dit que le nombre $r$ est la longueur véritable ou, simplement, la longuer de la chaîne. [19].

1bis) En anglais ,,descending chain condition" resp. ,,ascending chain condition"; voir 
si les conditions $\Omega \geqq \Omega^{\prime} \geqq a, \Omega \geqq \Omega^{\prime} \geqq b$ et $\omega \leqq \omega^{\prime} \leqq a, \omega \leqq \omega^{\prime} \leqq b$ entraînent (simultanément) $\Omega^{\prime}=\Omega$ et $\omega^{\prime}=\omega$; ainsi, par exemple, $S$ étant une structure, le quadrilatère $(a \vee b, a \wedge b ; a, b)$ y est toujours irréductible. Cette notion de quadrilatère irréductible va jouer dans la seconde partie de ce mémoire un rôle primordial.

1.4. Connexions monotones. Soient $P, P^{\prime}$ deux ensembles (distincts ou non) partiellement ordonnés arbitraires. Je dis que ces ensembles sont reliés par une connexion monotone s'il y existe une opération $\chi$ qui associe à tout $p \in P$ une partie non vide $\chi(p)$ de $P^{\prime}$ et une opération $\varphi$ qui associe à tout $p^{\prime} \in P^{\prime}$ une partie non vide $\varphi\left(p^{\prime}\right)$ de $P$, de telle façon que ces opérations $\chi$, $\varphi$ satisfassent aux axiomes suivants:

A. Pour chaque paire $\left(p_{1} \geqq p_{2}\right) \in P$ et chaque $q_{2}^{\prime} \in \chi\left(p_{2}\right)$ il existe au moins un $q_{1}^{\prime} \in \chi\left(p_{1}\right)$ tel qu'on ait $q_{1}^{\prime} \geqq q_{2}^{\prime}$. Pour chaque paire $\left(p_{1}^{\prime} \geqq p_{2}^{\prime}\right) \epsilon P^{\prime}$ et chaque $q_{1} \in \varphi\left(p_{1}^{\prime}\right)$ il existe au moins $q_{2} \in \varphi\left(p_{2}^{\prime}\right)$ tel $q u^{\prime}$ on ait $q_{1} \geqq q_{2}$.

B. Pour chaque $p \in P$ et chaque $q^{\prime} \epsilon \chi(p)$ il y a au moins un $\bar{p} \epsilon \varphi\left(q^{\prime}\right)$ tel que $\bar{p} \leqq p$; pour chaque $p^{\prime} \in P^{\prime}$ et chaque $q \in \varphi\left(p^{\prime}\right)$ il y a au moins un $\bar{p}^{\prime} \in \chi(q)$ tel que $\bar{p}^{\prime} \geqq p^{\prime}$.

Supposons que les opérations $\chi, \varphi$ soient univoques, c'est-à-dire que, quels que soient $p \in P$ et $p^{\prime} \in P^{\prime}$, chacun des ensembles $\chi(p)$ et $\varphi\left(p^{\prime}\right)$ n'ait toujours qu'un seul élément; alors, les définitions $\boldsymbol{A}+\boldsymbol{B}$ ci-dessus se laissent simplifier comme suit:

$\mathbf{A}_{0}$. Pour chaque paire $\left(p_{1} \geqq p_{2}\right) \in P$ on a $\chi\left(p_{1}\right) \geqq \chi\left(p_{2}\right)$; de même, pour chaque paire $\left(p_{1}^{\prime} \geqq p_{2}^{\prime}\right) \in P^{\prime}$ on a $\varphi\left(p_{1}^{\prime}\right) \geqq \varphi\left(p_{2}^{\prime}\right)$.

$\boldsymbol{B}_{0}$. Pour chaque $p \in P$ on a $\varphi \chi(p) \leqq p$; pour chaque $p^{\prime} \in P^{\prime}$ on a $\chi \varphi\left(p^{\prime}\right) \geqq p^{\prime}$.

Ce sont ces dernières correspondances que j'ai appelées ailleurs [15], connexions monotones mixtes; quant aux connexions monotones de première et de seconde espèce [15], elles n'ont, vraisemblablement, qu'un intérêt purement formel et, c'est pourquoi j'ai omis de les mentionner ici.

Je vais indiquer par la notation

$$
P \rightleftarrows P^{\prime} \bmod \chi, \varphi
$$

le fait que les ensembles partiellement ordonnés $P, P^{\prime}$ sont reliés par une connexion monotone moyennant les opérations (en général, multivoques) $\chi, \varphi$. La relation (1) n'est pas, en général, symétrique par rapport à $P$ et $P^{\prime}$ (même dans le cas où $\chi, \varphi$ sont univoques).

Je dis que la connexion monotone (1) est parfaite dans $P$ et j'écris

$$
P \longleftrightarrow P^{\prime} \bmod \chi, \varphi,
$$

lorsque pour chaque $p \in P$ et chaque $q^{\prime} \in \chi(p)$, on peut trouver, parmi tous les $\bar{p} \in \varphi\left(q^{\prime}\right)$ tels que $\bar{p} \leqq p$, au moins un, soit $\bar{p}_{0}$, tel que $\bar{p}_{0}=p$.

Pareillement, je dis que la connexion monotone (1) est parfaite dans $P^{\prime}$, et j'écris

$$
P \rightleftarrows P^{\prime} \bmod \chi, \varphi,
$$


lorsque pour chaque $p^{\prime} \in P^{\prime}$ et chaque $q \epsilon \varphi\left(p^{\prime}\right)$, on peut trouver, parmi tous les $\bar{p}^{\prime} \in \chi(q)$ tels que $\bar{p}^{\prime} \geqq p^{\prime}$, au moins un, soit $\bar{p}_{0}^{\prime}$, tel que $\bar{p}_{0}^{\prime}=p^{\prime}$.

Enfin, je dis que la connexion monotone (1) est parfaite, si elle est $\dot{a}$ la fois parfaite dans $P$ et dans $P^{\prime}$ et j'écris dans ce cas

$$
P \longleftrightarrow P^{\prime} \bmod \chi, \varphi
$$

Remarquons que dans le cas où les opérations $\chi, \varphi$ sont univoques, cette relation (2) signifie qu'il y a isomorphisme entre les deux ensembles partiellement ordonnés $P$ et $P^{\prime}$.

1.5. Composition des connexions monotones. Soient $P, P^{\prime}, P^{\prime \prime}$ trois ensembles partiellement ordonnés (distincts ou non) et supposons qu'on ait les relations

$$
P \rightleftarrows P^{\prime} \bmod \chi, \varphi ; \quad P^{\prime} \rightleftarrows P^{\prime \prime} \bmod \chi^{\prime}, \varphi^{\prime} .
$$

Composer les connexions (3) c'est, par définition, instituer entre $P$ et $P^{\prime \prime}$ la correspondance définie par les équations suivantes

$$
\chi^{\prime} \chi(p)=\sigma(p), p \in P ; \quad \varphi \varphi^{\prime}\left(p^{\prime \prime}\right)=\tau\left(p^{\prime \prime}\right), p^{\prime \prime} \in P^{\prime \prime} .
$$

Lemme. On a $P \rightleftarrows P^{\prime \prime} \bmod \sigma, \tau$.

La vérification en est immédiate.

Remarque. Pour les propriétés générales des connexions monotones (univoques), voir mon mémoire [15].

\section{§ 2. LES CONNEXIONS MONOTONES D'UN ENSEMBLE PARTIELLEMENT ORDONNÉ, ESPACES 1}

Dans les deux paragraphes suivants, nous exposerons les principes d'une méthode générale pour l'étude des ensembles partiellement ordonnés (arbitraires); cette méthode, je l'ai d'ailleurs déjà utilisée dans la théorie des structures à propos du problème des décompositions du type JORDAN-HöLDER $[16,17]$.

Elle consiste essentiellement en ce qu'un ensemble partiellement ordonné y est conçu comme un ,espace" dont certaines parties sont reliées par des systèmes de connexions monotones; c'est ici que la notion de quadrilatère de Dedekind viendra jouer un role principal.

Les propriétés d'un ensemble partiellement ordonné seront donc, dans cette théorie, les propriétés des systèmes de connexions dont il est muni, ainsi que de leurs relations mutuelles; telle propriété est, par exemple, le théorème de raffinement de Schreier (§ 3).

2.1. Espaces $\Delta$. Soient $P$ un ensemble partiellement ordonné et $(\Omega, \omega ; a, b)$ un de ses quadrilatères à côtés opposés semblables; nous ferons correspondre aux quotients $\Omega / a, b / \omega$ un ensemble $\Gamma$ de paires d'opérations $(\chi, \varphi)$ et aux quotients $\Omega / b, a / \omega$ un ensemble $\tilde{\Gamma}$ de paires d'opérations $(\tilde{\chi}, \tilde{\varphi})$ de façon que 
pour toute paire $(\chi, \varphi) \epsilon \Gamma$ et pour toute paire $(\tilde{\chi}, \tilde{\varphi}) \epsilon \Gamma$ on ait respectivement (au sens des axiomes $\boldsymbol{A}+\mathbf{B}$ ou $\boldsymbol{A}_{\mathbf{0}}+\boldsymbol{B}_{\mathbf{0}}, \mathbf{1 , 4}$ )

$$
\left.\Omega / a \rightleftarrows b / \omega \bmod \chi, \varphi ; \quad \Omega / b \rightleftarrows a / \omega \bmod \tilde{\chi}, \tilde{\varphi} \cdot{ }^{2}\right)
$$

Nous supposons en outre que les systèmes $\Gamma, \tilde{\Gamma}$ satisfont aux conditions suivantes:

A*. Pour toute paire $(\chi, \varphi) \in \Gamma$ il existe un $p_{a}^{\prime} \in \chi(a)$ et un $p_{b} \in \varphi(b)$ tels que $p_{a}^{\prime}=\omega$ et $p_{b}=\Omega$.

B*. Pour toute paire $(\tilde{\chi}, \tilde{\varphi}) \in \Gamma$ il existe un $q_{b}^{\prime} \in \tilde{\chi}(b)$ et un $q_{a} \in \tilde{\varphi}(a)$ tels que $q_{b}^{\prime}=\omega$ et $q_{a}=\Omega$.

$F^{*}$. Les systèmes $\Gamma, \tilde{\Gamma}$ sont maximaux par rapport aux propriétés $A^{*}, B^{*}$ respectivement.

Dans le cas où les opérations $(\chi, \varphi),(\tilde{\chi}, \tilde{\varphi})$ sont univoques, les conditions $A^{*}+B^{*}$ s'énoncent simplement:

$\boldsymbol{A}_{0}^{*}$. Pour toute paire $(\chi, \varphi) \epsilon \Gamma_{0}$ on a $\chi(a)=\omega, \varphi(b)=\Omega$.

$\mathbf{B}_{\mathbf{0}}^{*}$. Pour toute paire $(\tilde{\chi}, \tilde{\varphi}) \epsilon \tilde{\Gamma}_{\mathbf{0}}$ on a $\tilde{\chi}(b)=\omega, \tilde{\varphi}(a)=\Omega$.

Ici, on a désigné par $\Gamma_{0}$ la partie de $\Gamma$, formée par les paires d'opérations univoques; de même pour $\tilde{\Gamma}_{0}$ par rapport à $\tilde{\Gamma}$.

Un ensemble partiellement ordonné $P$ dont tous les quadrilatères $(\Omega, \omega$; $a, b)$ à côtés opposés semblables ont été munis de systèmes de connexions monotones $\Gamma, \tilde{\Gamma}$ satisfaisant à (4) et aux suppositions $A^{*}+B^{*}+\Gamma^{*}$, sera dit un espace $\Delta$.

2.11. A ce point, je fais remarquer qu'en vertu de la supposition $\Gamma^{*}$, les systèmes $\Gamma_{0}, \tilde{\Gamma}_{0}$ (a fortiori $\Gamma, \tilde{\Gamma}$ ) ne sont jamais vides. On peut, en effet, quel que soit le quadrilatère à côtés opposés semblables $(\Omega, \omega ; a, b) \in P$, définir $\chi_{\mathrm{o}}(\Omega)=b$ et $\chi_{\mathrm{o}}(p)=\omega$ pour chaque $p$ tel que $\Omega>p \geqq a$ (s'il en existe), $\varphi_{\mathrm{o}}(\omega)=a$ et $\varphi_{\mathrm{o}}\left(p^{\prime}\right)=\Omega$ pour chaque $p^{\prime}$ tel que $b \geqq p^{\prime}>\omega$ (s'il en existe), $\}$ $\tilde{\chi}_{o}(\Omega)=a$ et $\tilde{\chi}_{o}(q)=\omega$ pour chaque $q$ tel que $\Omega>q \geqq b$ (s'il en existe), $\tilde{\varphi}_{\mathrm{o}}(\omega)=b$ et $\tilde{\varphi}_{\mathrm{o}}\left(q^{\prime}\right)=\Omega$ pour chaque $q^{\prime}$ tel que $a \geqq q^{\prime}>\omega$ (s'il en existe). $\}$

On vérifie tout de suite qu'on a

$$
\left.\Omega / a \rightleftarrows b / \omega \bmod \chi_{o}, \varphi_{o} ; \quad \Omega / b \rightleftarrows a / \omega \bmod \tilde{\chi}_{o}, \tilde{\varphi}_{o}^{2}\right)
$$

et que les couples $\left(\chi_{0}, \varphi_{0}\right),\left(\tilde{\chi}_{0}, \tilde{\varphi}_{0}\right)$ satisfont, en outre, aux suppositions $\boldsymbol{A}_{0}^{*}$ et

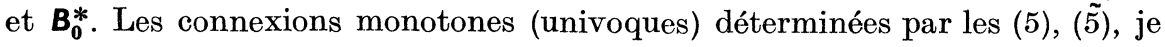
les ai appelées connexions de Ore associées au quadrilatère $(\Omega, \omega ; a, b)$; la raison de cette dénomination sera expliquée un peu plus loin.

Parce que les systèmes $\Gamma_{0}, \tilde{\Gamma}_{0}$ sont suffisants pour les besoins de la majeure partie de ce mémoire, ce sont ces systèmes que nous allons notamment considérer dans ce qui suit; quant aux systèmes $\Gamma, \tilde{\Gamma}$ et, plus particulièrement à la

${ }^{2}$ ) C'est-à-dire que les paires de quotients $\Omega / a, b / \omega$ et $\Omega / b, a / \omega$ y jouent, tour à tour, le rôle de la paire $P, P^{\prime}$ dans les axiomes mentionnés. 
connexion de Dedekind multivoque, bien que nous les utilisons dans ce mémoire (seconde partie) nous n'en ferons pas ici une étude détaillée. ${ }^{3}$ )

2.2. Remarques sur les connexions de 0re. Soient $P$ un espace $\Delta,(\Omega, \omega$; $a, b)$ un de ses quadrilatères à côtés opposés semblables et $\Gamma_{0}, \tilde{\Gamma_{0}}$ les systèmes de connexions univoques qui lui sont associés: $\Gamma_{0}$ au couple $\Omega / a, b / \omega$ et $\tilde{\Gamma}_{0}$ au couple $\Omega / b, a / \omega$. Alors on a pour $(\chi, \varphi) \epsilon \Gamma_{0}$, et $(\tilde{\chi}, \tilde{\varphi}) \epsilon \tilde{\Gamma}_{0}$,

$$
\chi_{0} \leqq \chi, \quad \varphi_{0} \geqq \varphi ; \quad \tilde{\chi}_{0} \leqq \tilde{\chi}, \quad \tilde{\varphi}_{0} \geqq \tilde{\varphi}
$$

où $\left(\chi_{0}, \varphi_{0}\right) \in \Gamma_{0}$ et $\left(\tilde{\chi}_{o}, \tilde{\varphi}_{0}\right) \in \tilde{\Gamma}_{0}$ sont les connexions de Ore du quadrilatère $(\Omega, \omega ; a, b)$ et où $\chi_{0} \leqq \chi$, etc., signifie, comme à l'ordinaire, que $\chi_{o}(p) \leqq \chi(p)$ pour tout $p \in \Omega / a$, etc.

Pour démontrer les (6) il suffit de remarquer que la connexion univoque ${ }^{4}$ ) $\Omega / a \rightleftarrows b / \omega \bmod \chi, \varphi$, entraîne

$$
\chi(\Omega)=b=\chi \varphi(b) ; \quad \varphi(\omega)=a=\varphi \chi(a) .
$$

En effet, on a successivement: $\Omega \geqq \varphi(b) \geqq a, b \geqq \chi(\Omega) \geqq \chi \varphi(b) \geqq b$; donc $\chi(\Omega)=b=\chi \varphi(b)$ etc.

2.21. Déterminons les ensembles des éléments parfaits ${ }^{5}$ ) des connexions de Ore de $(\Omega, \omega ; a, b)$.

Nous écartons évidemment le cas trivial où tous les côtés de ce quadrilatère sont nuls simultanément; supposons donc $\Omega>a$ et aussi, par suite, $b>\omega$. D'après les définitions (5), on aura alors:

$$
\varphi_{0} \chi_{0}(\Omega)=\Omega, \quad \varphi_{0} \chi_{0}(a)=a ; \quad \chi_{0} \varphi_{0}(b)=b, \quad \chi_{0} \varphi_{0}(\omega)=\omega .
$$

Mais s'il existe un $p \in \Omega / a$ tel que $\Omega>p>a$, alors $\varphi_{0} \chi_{0}(p) \leqq p$ signifie toujours $\varphi_{o} \chi_{o}(p)<p$, car on a $\chi_{0}(p)=\omega$ et $\varphi_{o}(\omega)=a$; de même, s'il existe $p^{\prime} \in b / \omega$ tel que $b>p^{\prime}>\omega$, alors on a $\chi_{0} \varphi_{0}\left(p^{\prime}\right)>p^{\prime}$.

Ainsi, les ensembles d'éléments parfaits de la connexion $\Omega / a \rightleftarrows b / \omega \bmod \chi_{0}, \varphi_{o}$, sont respectivement: $\{\Omega, a\}$ pour $\Omega / a$ et $\{b, \omega\}$ pour $b / \omega$. Conclusion analogue concernant la connexion $\Omega / b \rightleftarrows a / \omega \bmod \tilde{\chi}_{0}, \tilde{\varphi}_{0}$.

2.22. Réciproquement, cette propriété des connexions de Ore, suffit pour les caractériser.

Supposons en effet qu'une connexion $(\chi, \varphi) \epsilon \Gamma_{0}$ soit telle que les équations

$$
\varphi \chi(p)=p, \quad p \in \Omega / a ; \quad \chi \varphi\left(p^{\prime}\right)=p^{\prime}, \quad p^{\prime} \epsilon b / \omega
$$

n'aient d'autres solutions que $p=\Omega, a$ et $p^{\prime}=b, \omega$ respectivement; alors,

3) Laquelle me paraît, du reste, devoir être précédée par une théorie générale des correspondances multivoques.

4) Cela ne signifie pas qu'on ait $(\chi, \varphi) \in \Gamma_{0}$, car la supposition $\boldsymbol{A}_{0}^{*}$ peut ne pas y être satisfaite.

$\left.{ }^{5}\right)$ Soit $P \rightleftarrows P^{\prime} \bmod \chi, \varphi$; je dis que $p \in P$ est parfait lorsque $\varphi \chi(p)=p$ et, pareillement, $p^{\prime} \in P^{\prime}$ est parfait lorsque $\chi \varphi\left(p^{\prime}\right)=p^{\prime}$. 
je dis que $\chi=\chi_{0}$ et $\varphi=\varphi_{0}$. En effet, on a d'abord, par les $(7), \chi(\Omega)=b$, puis, par la $A_{0}^{*}(2.1), \chi(a)=\omega$.

Supposons donc, s'il y a lieu, que $p \in \Omega / a$ soit tel que $\Omega>p>a$; on en déduit $\chi(\Omega) \geqq \chi(p) \geqq \chi(a)$, c'est-à-dire $b \geqq \chi(p) \geqq \omega$. Or, pour chaque $p \in \Omega / a$, on a $\chi \varphi, \chi(p)=\chi(p)[15]$; on aura donc, en vertu de l'hypothèse, $\chi(p)=b, \omega$. Mais, $\chi(p)=b$ est impossible car $p \geqq \varphi \chi(p)=\varphi(b)=\Omega$; par conséquent $\chi(p)=\omega$. Ainsi, on a bien $\chi(p)=\chi_{0}(p)$, pour tout $p \epsilon \Omega / a$ et on verrait de même que $\varphi=\varphi_{0}$.

2.3. Connexions radiales. Soit $(\Omega, \omega ; a, b)$ un quadrilatère à côtés opposés semblables et soit $\left.\Omega / a \rightleftarrows b / \omega \bmod \chi, \varphi^{4}\right)$; je dis que cette connexion est radiale lorsque l'une, au moins, des deux conditions suivantes y est remplie: $R_{1}$. Pour tout $p \in \Omega / a$ on a $\chi(p) \leqq p, R_{2}$. Pour tout $p^{\prime} \epsilon b / \omega$ on a $\varphi\left(p^{\prime}\right) \geqq p^{\prime}$.

Lemme. Pour que la condition $R_{1}$ soit remplie il faut et il suffit que pour tout $p \in \Omega / a$ tel que $\varphi \chi(p)=p$ on ait $\chi(p) \leqq p$. Dans ce cas, la condition $R_{2} y$ est aussi remplie.

Démonstration. La condition énoncée est évidemment nécessaire; mais cette condition est aussi suffisante, car on a successivement: $\left(p^{\prime} \epsilon b / \omega\right), \varphi \chi$. . $\varphi\left(p^{\prime}\right)=\varphi\left(p^{\prime}\right)[14], p^{\prime} \leqq \chi \varphi\left(p^{\prime}\right) \leqq \varphi\left(p^{\prime}\right)$, donc $\varphi\left(p^{\prime}\right) \geqq p^{\prime}\left(p^{\prime} \in b / \omega !\right)$ (c'est la seconde partie de l'énoncé); par conséquent $\varphi \chi(p) \geqq \chi(p)$ pour chaque $p \in \Omega / a$ et comme $\varphi \chi(p) \leqq p$ il en résulte finalement $\chi(p) \leqq p$ avec $p \in \Omega / a, \mathrm{Q}$. E. D.

Remarquons que les connexions de Ore sont des connexions radiales; mais, dans un espace $\Delta$, il peut y avoir des quadrilatères $(\Omega, \omega ; a, b)$ dont les seules connexions radiales $(\chi, \varphi) \in \Gamma_{0}(\Omega / a, b / \omega)$ et $(\tilde{\chi}, \tilde{\varphi}) \in \tilde{\Gamma_{0}}(\Omega / b, a / \omega)$ soient les connexions de Ore. Tels sont, par exemple, les quadrilatères $(\Omega, \omega ; a, b)$ qu'on peut former en prenant pour $\Omega, \omega$ les extrémités d'un cycle simple $\left(C_{1}, C_{2}\right)$, [11], et pour $a, b$ des éléments tels que $a \epsilon C_{1}, b \in C_{2}$ et tels que $a, b \neq \Omega, \omega$.

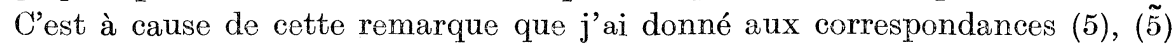
la dénomination de , connexions de Ore".

2.4. Les structures comme espaces 4 . Soit $S$ une structure arbitraire et $(\Omega, \omega ; a, b)$ un de ses quadrilatères (à côtés opposés semblables). Posons:

$$
\begin{array}{cc}
\chi_{D}^{*}(p)=b \wedge p, \quad p \in \Omega / a ; & \varphi_{D}^{*}\left(p^{\prime}\right)=a \vee p^{\prime}, \quad p^{\prime} \in b / \omega, \\
\tilde{\chi}_{D}^{*}(q)=a \wedge q, \quad q \in \Omega / b ; & \tilde{\varphi}_{D}^{*}\left(q^{\prime}\right)=b \vee q^{\prime}, q^{\prime} \in a / \omega .
\end{array}
$$

On vérifie immédiatement les relations

$$
\Omega / a \rightleftarrows b / \omega \bmod \chi_{D}^{*}, \varphi_{D}^{*} ; \quad \Omega / b \rightleftarrows a / \omega \bmod \tilde{\chi}_{D}^{*}, \tilde{\varphi}_{D}^{*} .
$$

On a en général $\left(\chi_{D}^{*}, \varphi_{D}^{*}\right)$ non $\epsilon \Gamma_{0}(\Omega / a, b / \omega),\left(\tilde{\chi}_{D}^{*}, \tilde{\varphi}_{D}^{*}\right)$ non $\epsilon \tilde{\Gamma}_{0}(\Omega / b, a / \omega)$, à moins

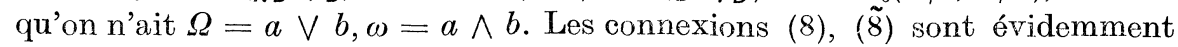
radiales.

Les opérations univoques $\left(\chi_{D}, \varphi_{D}\right),\left(\tilde{\chi}_{D}, \tilde{\varphi}_{D}\right)$ qu'on déduit de (8), (8) par la restriction des domaines de celles-ci aux côtés correspondants du sousquadrila- 
tère $(a \vee b, a \wedge b ; a, b)$, sont ce que j'ai appelé les connexions de Dedekind (associées à ce sousquadrilatère).

Désignons par $\left(\chi_{0}, \varphi_{0}\right) \in \Gamma_{0}(a \vee b / a, b / a \wedge b),\left(\tilde{\chi}_{0}, \tilde{\varphi}_{0}\right) \epsilon \tilde{\Gamma}_{0}(a \vee b / b, a / a \wedge b)$ les connexions de Ore attachées à $(a \vee b, a \wedge b ; a, b)$; on peut écrire, évidemment:

$$
\begin{aligned}
& \chi_{0} \leqq \chi_{D}, \quad \varphi_{0} \geqq \varphi_{D}, \\
& \tilde{\chi}_{0} \leqq \tilde{\chi}_{D}, \quad \tilde{\varphi}_{O} \geqq \tilde{\varphi}_{D}
\end{aligned}
$$

et si $(\chi, \varphi) \epsilon \Gamma_{0}(a \vee b / a, b / a \wedge b),(\tilde{\chi}, \tilde{\varphi}) \epsilon$ $\epsilon \tilde{\Gamma}_{0}((a \vee b / b, a / a \wedge b) \mathrm{s}$ ont des connexions radiales arbitraires, on aura encore

$$
\begin{aligned}
& \chi_{0} \leqq \chi \leqq \chi_{D}, \quad \varphi_{O} \geqq \varphi \geqq \varphi_{D}, \\
& \tilde{\chi}_{0} \leqq \tilde{\chi} \leqq \tilde{\chi}_{D}, \quad \tilde{\varphi}_{O} \geqq \tilde{\varphi} \geqq \tilde{\varphi}_{D},
\end{aligned}
$$

c'est-à-dire que $\chi(p) \leqq \chi_{D}(p), p \in a \vee b / a$ puis $\varphi\left(p^{\prime}\right) \geqq \varphi_{D}\left(p^{\prime}\right), p^{\prime} \in b / a \wedge b$, etc.

Des exemples bien simples, tels que le suivant, montrent l'existence dans (10) ou ( $\tilde{10})$ de connexions monotones (radiales) $(\chi, \varphi)$ ou $(\tilde{\chi}, \tilde{\varphi})$ distinctes et des connexions de Ore et des connexions de Dedekind.

2.41. Exemple. Considérons le schéma de

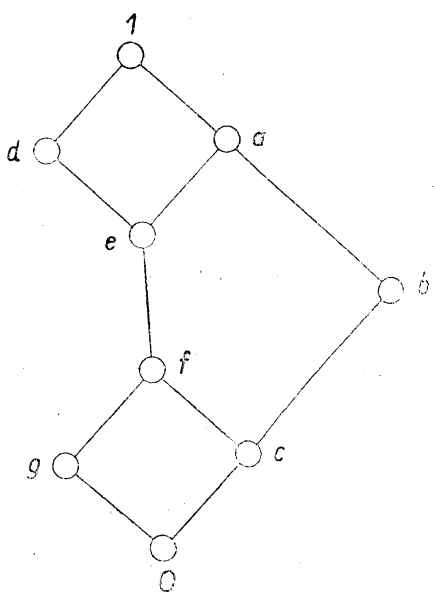

Fig. 1. structure ci-contre (Fig. 1).

Le quadrilatère $(1, c ; b, d)$ y est à côtés opposés semblables et l'on a $b \vee d=1$, $b \wedge d=c$. Désignons, comme à l'ordinaire, $\operatorname{par}\left(\chi_{0}, \varphi_{0}\right)$ et $\left(\chi_{D}, \varphi_{D}\right)$ les connexions de Ore et de Dedekind, reliant $1 / b$ à $d / c$ et par $\left(\tilde{\chi}_{O}, \tilde{\varphi}_{O}\right),\left(\tilde{\chi}_{D}, \tilde{\varphi}_{D}\right)$ les connexions de mêmes noms entre $1 / d$ et $b / c$. On a évidemment

Or, si l'on pose:

$$
\tilde{\chi}_{O}=\tilde{\chi}_{D}, \quad \tilde{\varphi}_{O}=\tilde{\varphi}_{D} .
$$

$$
\left.\begin{array}{l}
\chi(1)=d, \chi(a)=f, \chi(b)=c, \\
\varphi(d)=1, \varphi(e)=1, \varphi(f)=a, \varphi(c)=b
\end{array}\right\}
$$

la correspondance ainsi obtenue, entre les quotients $1 / b$ et $d / c$, est, on s'en convainc facilement, une connexion monotone radiale: $\left.1 / b \rightleftarrows d / c \bmod \chi, \varphi^{6}\right)$; de plus $(\chi, \varphi) \in \Gamma_{0}(1 / b, d / c)$. On a donc

$$
\chi_{0} \leqq \chi \leqq \chi_{D}, \quad \varphi_{O} \geqq \varphi \geqq \varphi_{D} .
$$

Mais $\chi_{\mathrm{O}}(a)=c, \chi(a)=f$ et $\chi_{\mathrm{D}}(a)=e$; par conséquent, $\chi_{\mathrm{O}}<\chi<\chi_{\mathrm{D}}$. Puis on a $\varphi_{O}(f)=1, \varphi(f)=a$ donc $\varphi_{O}>\varphi$; et $\varphi(e)=1, \varphi_{D}(e)=a$ donc $\varphi>\varphi_{D}$. On aura done finalement $\varphi_{O}>\varphi>\varphi_{D}$.

$\left.{ }^{6}\right)$ On peut même écrire $1 / b \rightleftarrows d / c \bmod \chi, \varphi$. 
2.5. Supposition $\boldsymbol{H}$ et espaces $\Delta$ modulaires. Soit $\boldsymbol{P}$ un espace $\Delta$; je dis que $\boldsymbol{P}$ satisfait à la supposition $\boldsymbol{H}$, s'il existe au moins un $\omega_{0} \in P$ tel que dans tout quadrilatère $\left(\Omega, \omega_{0} ; a, b\right)$ on puisse trouver un sousquadrilatère $(M, d$; $a, b)$ à côtés opposés semblables, de façon que les systèmes $\Gamma_{0}(M / a, b / d), \tilde{\Gamma}_{0}(M / b$, a/d) jouissent de la propriété suivante:

$\boldsymbol{H}$. Il existe une connexion $(\chi, \varphi) \epsilon \Gamma_{\mathbf{0}}(M / a, b / d)$ et une connexion $(\tilde{\chi}, \tilde{\varphi}) \epsilon$ $\epsilon \tilde{\Gamma_{0}}(M / b, a / d)$ telles qu'on ait

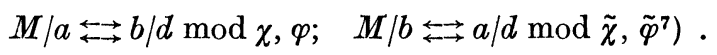

On ne suppose dans cet énoncé ni que le quadrilatère $(M, d ; a, b)$ soit irréductible ni que les connexions (13) soient radiales.

Nous dirons que $P$ est un espace $\Delta$ modulaire s'il vérifie la supposition $\boldsymbol{H}$, quel que soit $\omega_{0} \epsilon P$.

\section{§ 3. THÉOREME DE SCHREIER ET THÉORIE DES RAFFINEMENTS.}

Le problème que nous allons examiner dans ce paragraphe, se laisse énoncer comme suit:

Etant données, dans un ensemble partiellement ordonné $P$, deux chaînes aux extrémités communes, trouver un raffinement de la première chaîne et un raffinement de la seconde de telle façon, que les nouvelles châ̂nes aient même longueur et que leurs quotients soient (de chaîne à chaîne) isomorphes dans un certain ordre. ${ }^{8}$ )

Nous allons voir que si $P$ satisfait à la supposition $\boldsymbol{H}$, la réponse est toujours affirmative (au moins pour certaines paires de chaînes); nous en déduirons un procédé qui nous permettra de trouver de proche en proche les raffinements (mutuels) canoniques de deux chaînes aux extrémités communes.

3.1. Théorème de Schreier. Soit $\boldsymbol{P}$ un espace $\Delta$ satisfaisant à la supposition $\boldsymbol{H}$; alors, deux châ̂nes aux extrémités communes $\Omega \geqq \omega_{0}$ possèdent des raffinements isomorphes. (Ici, $\omega_{0}$ est un de ces éléments $\omega_{0}$ figurant dans l'énoncé de la supposition $\boldsymbol{H}$ de 2.5).

Première démonstration. (Le principe de cette démonstration est dû à Schreier lui-même (l. c.)).

Nous remarquons d'abord que, les chaînes $C_{1}, C_{2}$ ayant même longueur et des quotients isomorphes en quelque ordre (symbole: $C_{1} \cong C_{2}$ ), on peut faire correspondre à tout raffinement $C_{1}^{\prime}$ de $C_{1}$ un raffinement $C_{2}^{\prime}$ de $C_{2}$ de telle façon qu'on ait $C_{1}^{\prime} \cong C_{2}^{\prime}$.

7) Remarquons, à propos de cette définition, que, quels que soient l'espace $P$ et le quadrilatère $(\Omega, \omega ; a, b)$ avec $b \geqq a$, on peut $\mathrm{y}$ toujours trouver un sousquadrilatère $(M, d ; a, b)$ à côtés opposés semblables de façon que les (13) soient (trivialement) véri. fiées; il suffit de poser $M=b, d=a$.

$\left.{ }^{8}\right)$ De pareils raffinements, je les appellerai, pour abréger, raffinements isomorphes. 
Cela étant, pour démontrer que les chaînes

$$
\left.\begin{array}{l}
\Omega=a_{0} \geqq a_{1} \geqq \ldots \geqq a_{i-1} \geqq a_{i} \geqq \ldots \geqq a_{r-1} \geqq a_{r}=\omega_{0} \\
\Omega=b_{0} \geqq b_{1} \geqq \ldots \geqq b_{j-1} \geqq b_{j} \geqq \ldots \geqq b_{s-1} \geqq b_{s}=\omega_{0}
\end{array}\right\}
$$

possèdent des raffinements isomorphes, nous allons utiliser une double induction. Le théorème est évidemment vrai pour $r=s=2$, attendu qu'on a posé $\Omega / M \rightleftarrows \Omega / M$ et $d / \omega_{0} \leftrightarrows d / \omega_{0}$ (voir 2.5).

Supposons donc que le théorème soit vrai pour chaque paire de chaînes

$$
\begin{gathered}
\Omega^{\prime}=a_{0}^{\prime} \geqq a_{1}^{\prime} \geqq \ldots \geqq a_{r-1}^{\prime}=\omega_{0} \\
\Omega^{\prime}=b_{0}^{\prime} \geqq b_{1}^{\prime} \geqq b_{2}^{\prime}=\omega_{0}
\end{gathered}
$$

et démontrons-le pour les paires (14) avec $s=2$. Or, puisqu'on a $\Omega \geqq a_{1} \geqq$ $\geqq \omega_{0}$ et $\Omega \geqq b_{1} \geqq \omega_{0}$, il résulte de la supposition $\boldsymbol{H}$ l'existence d'une paire $M, d \in P$ telle que $\Omega \geqq M \geqq a_{1} \geqq d \geqq \omega_{0}$ et $\Omega \geqq M \geqq b_{1} \geqq d \geqq \omega_{0}$ et telle que

$$
M / a_{1} \leftrightarrow b_{1} / d \bmod \chi, \varphi ; \quad M / b_{1} \leftrightarrow a_{1} / d \bmod \tilde{\chi}, \tilde{\varphi}
$$

On peut donc écrire

$$
\left(M \geqq a_{1} \geqq d \geqq \omega_{0}\right) \cong\left(M \geqq b_{1} \geqq d \geqq \omega_{0}\right) .
$$

Mais les chaînes $a_{1} \geqq a_{2} \geqq \ldots \geqq a_{r-1} \geqq a_{r}=\omega_{0}$ et $a_{1} \geqq d \geqq \omega_{0}$ possèdent, d'après l'hypothèse inductive des raffinements isomorphes

$$
\left(a_{1} \geqq \ldots \geqq a_{2} \geqq \ldots \geqq \ldots \geqq \omega_{0}\right) \cong\left(a_{1} \geqq \ldots \geqq d \geqq \ldots \geqq \omega_{0}\right) .
$$

Or, le second membre de $\left(15^{\prime}\right)$ engendre un raffinement du premier membre de (15); et à ce raffinement on peut, vu la remarque ci-dessus, faire correspondre dans le second membre de (15) un autre, qui lui soit isomorphe. On aura donc

$$
\left(M \geqq a_{1} \geqq \ldots \geqq d \geqq \ldots \geqq \omega_{0}\right) \cong\left(M \geqq \ldots \geqq b_{1} \geqq d \geqq \ldots \geqq \omega_{0}\right) .
$$

Les $\left(15^{\prime}\right)$ et $\left(15^{\prime \prime}\right)$ donnent alors (grâce à la transitivité de $\cong$ )

$$
\left(\Omega \geqq M \geqq a_{1} \geqq \ldots \geqq a_{2} \geqq \ldots \geqq \ldots \geqq \omega_{0}\right) \cong\left(\Omega \geqq M \geqq \ldots \geqq b_{1} \geqq d \geqq\right.
$$

ce qui achève la démonstration de la première induction.

Supposons maintenant que le théorème soit vrai pour chaque paire de chaînes

$$
\Omega^{\prime}=a_{0}^{\prime} \geqq a_{1}^{\prime} \geqq \ldots \geqq a_{r}^{\prime}=\omega_{0}, \quad \Omega^{\prime}=b_{1}^{\prime} \geqq b_{2}^{\prime} \geqq \ldots \geqq b_{s-1}^{\prime} \geqq \omega_{0}
$$

et démontrons-le pour les paires (14). Or, les chaînes $a_{0} \geqq a_{1} \geqq \ldots \geqq a_{r}$ et $b_{0} \geqq b_{1} \geqq b_{s}$ possèdent, d'après la première partie de la démonstration, des raffinements isomorphes

$$
\begin{aligned}
& \left(\Omega \geqq \ldots \geqq a_{1} \geqq \ldots \geqq a_{2} \geqq \ldots \geqq \ldots \geqq \omega_{0}\right) \cong\left(\Omega \geqq \ldots \geqq b_{1} \geqq \ldots \geqq\right. \\
& \left.\geqq \omega_{0}\right) \text {. }
\end{aligned}
$$


Mais, la chaîne $b_{1} \geqq \ldots \geqq \omega_{0}$ (extraite du second membre de (16)) et la chaîne $b_{1} \geqq b_{2} \geqq \ldots \geqq b_{s}=\omega_{0}$ possèdent, elles aussi, en vertu de la nouvelle hypothèse inductive des raffinements isomorphes:

$$
\left(b_{1} \geqq \ldots \geqq \ldots \geqq \ldots \geqq \omega_{0}\right) \cong\left(b_{1} \geqq \ldots \geqq b_{2} \geqq \ldots \geqq \ldots \geqq \omega_{0}\right) .
$$

De $(16)$ et $\left(16^{\prime}\right)$ il résulte, toujours à l'aide de la remarque déjà utilisée, qu'on a finalement

$$
\begin{gathered}
\left(\Omega \geqq \ldots \geqq \ldots \geqq a_{1} \geqq \ldots \geqq a_{2} \geqq \ldots \geqq \ldots \geqq \ldots \geqq \omega_{0}\right) \cong \\
\cong\left(\Omega \geqq \ldots \geqq b_{1} \geqq \ldots \geqq b_{2} \geqq \ldots \geqq \ldots \geqq \ldots \geqq \omega_{0}\right) .
\end{gathered}
$$

Le théorème est démontré.

3.11. Deuxième démonstration. Je considère en premier lieu les chaînes

$$
\Omega \geqq a_{1} \geqq \omega_{0}, \Omega \geqq b_{1} \geqq b_{2} \geqq \ldots \geqq b_{j-1} \geqq b_{j} \geqq \ldots \geqq \omega_{0}
$$

dont la première est une partie de la première chaîne (14). Or, d'après la supposition $\boldsymbol{H}$, le quadrilatère $\left(\Omega, \omega_{0} ; a_{1}, b_{1}\right)$ possède toujours un sousquadrilatère $\left(a_{11}, d_{1}^{1} ; a_{1}, b_{1}\right)$ tel qu'on ait:

$$
a_{11} / a_{1} \longleftrightarrow b_{1} / d_{1}^{1}, \bmod \chi_{11}, \varphi_{11} ; a_{11} / b_{1} \longleftrightarrow a_{1} / d_{1}^{1} \bmod \tilde{\chi}_{11}, \tilde{\varphi}_{11}
$$

Le quadrilatère $\left(b_{1}, \omega_{0} ; d_{1}^{1}, b_{2}\right)$ possède, à son tour, toujours en vertu de la supposition $\boldsymbol{H}$, un sousquadrilatère $\left(a_{12}^{1}, d_{2}^{1} ; d_{1}^{1}, b_{2}\right)$ tel qu'on ait

$$
a_{12}^{1} / d_{1}^{1} \longleftrightarrow b_{2} / d_{2}^{1} \bmod \chi_{12}, \varphi_{12} ; \quad a_{12}^{1} / b_{2} \longleftrightarrow d_{1}^{1} / d_{2}^{1} \bmod \tilde{\chi}_{12}, \tilde{\varphi}_{12}
$$

Le quadrilatère $\left(b_{2}, \omega_{0} ; d_{2}^{1}, b_{3}\right)$ possède, de même, un sousquadrilatère $\left(a_{13}^{2}\right.$, $\left.a_{3}^{\prime \prime} ; d_{2}^{\prime 1}, b_{3}\right)$ tel qu'on ait

$$
a_{13}^{2} / d_{2}^{1} \leftrightarrows b_{3} / d_{3}^{1} \bmod \chi_{13}, \varphi_{13} ; \quad a_{13}^{2} / b_{3} \leftrightarrows d_{2}^{1} / d_{3}^{1} \bmod \tilde{\chi}_{13}, \tilde{\varphi}_{13}
$$

et ainsi de suite. Il se forme, de la sorte, une chaîne

$$
a_{1}=d_{0}^{1} \geqq d_{1}^{1} \geqq d_{2}^{1} \geqq \ldots \geqq d_{j-1}^{1} \geqq d_{j}^{1} \geqq \ldots \geqq d_{s-1}^{1} \geqq d_{s}^{1}=\omega_{0},
$$

telle que, le terme $d_{j-1}^{1}$ y étant déjà déterminé, on obtienne le suivant, $d_{j}^{1}$, par la considération du quadrilatère $\left(b_{j-1}, \omega_{0} ; d_{j-1}^{1}, b_{j}\right)$ ainsi que de l'un de ses sousquadrilatères $\left(a_{1 j}^{j-1}, d_{j}^{1} ; d_{j-1}^{1}, b_{j}\right)$ satisfaisant à la supposition $\boldsymbol{H}$ :

$$
a_{1 j}^{j-1} / d_{j-1}^{1} \overleftrightarrow{\longleftrightarrow} b_{j} / a_{j}^{1} \bmod \chi_{1 j}, \varphi_{1 j} ; a_{1 j}^{j-1} / b_{j} \longleftrightarrow d_{j-1}^{1} / d_{j}^{1} \bmod \tilde{\chi}_{1 j}, \tilde{\varphi}_{1 j}
$$

Pour le dernier quadrilatère $\left(b_{s-1}, \omega_{0} ; d_{s-1}^{1}, b_{s}\right)$ on peut naturellement poser $a_{1 s}^{s-1}=d_{s-1}^{1}$ et $d_{s}^{1}=\omega_{0}$.

Or, on a pour chaque $j=1,2, \ldots, s, \varphi_{1 j}\left(b_{j}\right)=a_{1 j}^{j-1}, a_{11}^{0}=a_{11}$; mais évidemment, $a_{1 j}^{j-1} \epsilon b_{j-1} / d_{j-1}^{1}$ et, par conséquent, $\varphi_{1, j-1}\left(a_{1 j}^{j-1}\right)$ a un sens. Posons donc $\varphi_{1, j-1} \varphi_{1 j}\left(b_{j}\right)=a_{1_{j}}^{j-2}$ et, en général

$$
\varphi_{1, j-k} \varphi_{1, j-k+1} \ldots \varphi_{1, j-1} \varphi_{1 j}\left(b_{j}\right)=a_{1 j}^{j-k-1}, k=0,1,2, \ldots, j-1 ; j>1 .
$$

Pour $k=j-1$, cette formule nous donne

$$
\varphi_{11} \varphi_{12} \ldots \varphi_{1, j-1} \varphi_{1 j}\left(b_{j}\right)=a_{1 j}^{0}=a_{1 j}, \quad j=1,2, \ldots, s ; \quad a_{1 s}=a_{1}
$$


et on vérifie sans peine qu'on a $a_{1 j} \in \Omega / a_{1}$. D'ailleurs, $a_{1, j-1} \geqq a_{1 j}, j \geqq 1$, attendu qu'on a posé, pour $j=1, a_{10}=\Omega$; on a, en effet, $b_{j-1} \geqq a_{1 j}^{j-1}$, donc $\varphi_{1, j-1}\left(b_{j-1}\right) \geqq$ $\geqq \varphi_{1, j-1}\left(a_{1 j}^{j-1}\right), \varphi_{1, j-2} \varphi_{1, j-1}\left(b_{j-1}\right) \geqq \varphi_{1, j-2} \varphi_{1, j-1}\left(a_{1 j}^{j-1}\right)$, etc.

Nous obtenons ainsi la chaîne

$$
\Omega=a_{10} \geqq a_{11} \geqq a_{12} \geqq \ldots \geqq a_{1, j-1} \geqq a_{1 j} \geqq \ldots \geqq a_{1, s-1} \geqq a_{1 s}=a_{1}
$$

avec la propriété très importante qu'on vérifie facilement:

$$
\left.\begin{array}{c}
a_{1, j-1} / a_{1 j} \rightleftarrows b_{j-1} / a_{1 j}^{j-1} \bmod \chi_{1, j-1} \cdots \chi_{12} \chi_{11}, \varphi_{11} \varphi_{12} \ldots \varphi_{1, j-1}, \\
j=1,2, \ldots, s ; a_{10}=\Omega, a_{1 s}=a_{1}, a_{1 s}^{s-1}=d_{s-1}^{1}
\end{array}\right\}
$$

La chaîne (21) est, par définition, un raffinement canonique de l'intervalle $\Omega \geqq a_{1}$ moyennant la seconde chaîne (14) et les connexions $\left(\chi_{1 j}, \varphi_{1 j}\right), j=$ $=1,2, \ldots s$.

Je considère en second lieu, la chaîne

$$
a_{1} \geqq a_{2} \geqq \omega_{0}
$$

et la chaîne (19) et je leur fais jouer le rôle de la première et de la seconde chaîne (17), respectivement. J'obtiens de la sorte, par le même enchaînement d'opérations que précédemment, une chaîne

$$
a_{2}=d_{0}^{2} \geqq d_{1}^{2} \geqq d_{2}^{2} \geqq \ldots \geqq d_{j-1}^{2} \geqq d_{j}^{2} \geqq \ldots \geqq d_{s-1}^{2} \geqq d_{s}^{2}=\omega_{0}
$$

avec la propriété évidente

$$
d_{j}^{2} \leqq d_{j}^{1}, \quad j=0,1,2, \ldots, s
$$

et un raffinement canonique

$$
a_{1}=a_{20} \geqq a_{21} \geqq a_{22} \geqq \ldots \geqq a_{2, j-1} \geqq a_{2 j} \geqq \ldots \geqq a_{2, s-1} \geqq a_{2 s}=a_{2}
$$

avec la propriété

$$
\begin{gathered}
\varphi_{21} \varphi_{22} \ldots \varphi_{2, j-1} \varphi_{2 j}\left(d_{j}^{1}\right)=a_{2 j}^{0}=a_{2 j} \\
a_{2, j-1} / a_{2 j} \leftrightarrows d_{j-1}^{1} / a_{2 j}^{j-1} \bmod \chi_{2, j-1} \cdots \chi_{22} \chi_{21}, \varphi_{21} \varphi_{22} \ldots \varphi_{2, j-1} \\
j=1,2, \ldots, s, \quad a_{2 s}^{s-1}=d_{s-1}^{2},
\end{gathered}
$$

où tous les symboles ont à l'égard des chaînes (22) et (19), même signification que les symboles correspondants à l'égard des chaînes (17).

Après avoir itéré ce procédé $i-1$ fois $(i=1,2, \ldots)$ et que l'on ait ainsi construit la chaîne

$a_{i-1}=d_{0}^{i-1} \geqq d_{1}^{i-1} \geqq d_{2}^{i-1} \geqq \ldots \geqq d_{j-1}^{i-1} \geqq d_{j}^{i-1} \geqq \ldots \geqq d_{s-1}^{i-1} \geqq d_{s}^{i-1}=\omega_{0}$

et le raffinement canonique de $a_{i-2} \geqq a_{i-1}$, je considère la chaîne $a_{i-1} \geqq$ $\geqq a_{i} \geqq \omega_{0}$ et je lui applique à l'égard de (23) le même traitement que dans tous les cas précédents. Je parviens ainsi à la chaîne

$$
\left.\begin{array}{c}
a_{i}=d_{0}^{i} \leqq d_{1}^{i} \geqq d_{2}^{i} \geqq \ldots \geqq d_{j-1}^{i} \geqq d_{j}^{i} \geqq \ldots \geqq d_{s-1}^{i} \geqq d_{s}^{i}=\omega_{0}, \\
i=0,1, \ldots, r ; \quad d_{j}^{0}=b_{j}, \quad j=0,1,2, \ldots, s
\end{array}\right\}
$$


ayant la propriété

$$
\left.\begin{array}{c}
d_{j}^{i} \leqq d_{j}^{i-1}, \quad i=1,2, \ldots, r, j=0,1,2, \ldots, s ; \\
d_{j}^{r}=\omega_{0}, \quad j=0,1,2, \ldots, s
\end{array}\right\}
$$

et au raffinement canonique

$$
\left.\begin{array}{c}
a_{i-1}=a_{i 0} \geqq a_{i 1} \geqq a_{i 2} \geqq \ldots \geqq a_{i, j-1} \geqq a_{i j} \geqq \ldots \geqq a_{i, s-1} \geqq a_{i s}=a_{j} ; \\
i=1,2, \ldots, r, \quad a_{r j}=d_{j}^{r-1} ; \quad j=0,1,2, \ldots, s
\end{array}\right\}
$$

avec les formules générales

$$
\left.\begin{array}{c}
a_{i j}^{j-1} / d_{i-1}^{i} \leftrightarrows d_{j}^{i-1} / d_{j}^{i} \bmod \chi_{i j}, \varphi_{i j} ; \quad a_{i j}^{j-1} / d_{j}^{i-1} \leftrightarrows d_{j-1}^{i} / d_{j}^{i} \bmod \tilde{\chi}_{i j}, \tilde{\varphi}_{i j}, \\
\left.a_{r j}^{i-1}=d_{j}^{r-1}, \quad a_{i j}^{j-1} \leqq d_{j-1}^{i-1} ; \quad i=1,2, \ldots, r, \quad j=1,2, \ldots, s, \quad\right\} \\
\varphi_{i 1} \varphi_{i 2} \ldots \varphi_{i, j-1} \varphi_{i j}\left(d_{j}^{i-1}\right)=a_{i j}^{0}=a_{i j} ; \quad i=1,2, \ldots, r, j=1,2, \ldots, s \\
a_{i, j-1} / a_{i j} \leftrightarrows d_{j-1}^{i-1} / a_{i j}^{j-1} \bmod \chi_{i, j-1} \ldots \chi_{i 2} \chi_{i 1}, \varphi_{i 1} \varphi_{i 2} \ldots \varphi_{i, j-1}, \\
a_{i s}^{s-1}=d_{s-1}^{i}, \quad a_{0 s}^{s-1}=d_{s-1}^{0}, \\
i=1,2, \ldots, r, \quad j=1,2, \ldots, s .
\end{array}\right\}
$$

Il importe de remarquer que les égalités $d_{j}^{0}=b_{j}, d_{j}^{r}=\omega_{0}, a_{r j}=d_{j}^{r-1}, a_{r j}^{j-1}=$ $=d_{j}^{r-1}, a_{i s}^{s-1}=d_{s-1}^{i}, a_{0 s}^{s-1}=d_{s-1}^{0}$ dans $(24), \quad\left(24^{\prime}\right), \quad\left(24^{\prime \prime}\right), \quad(25), \quad\left(25^{\prime \prime}\right)$ sont des définitions.

On a d'ailleurs, en vertu des secondes relations (25) pour $i=r-1$ et avec les notations que nous avons adoptées

$$
\left.\begin{array}{c}
a_{r, j-1} / a_{r j} \rightleftarrows a_{r-1, j}^{j-1} / d_{j}^{r-2} \bmod \tilde{\varphi}_{r-1, j}, \tilde{\chi}_{r-1, j}, \\
j=1,2, \ldots, s .
\end{array}\right\}
$$

Nous avons par ce qui précède obtenu ce que j'appelerai le raffinement canonique de la première chaîne (14) moyennant la seconde chaîne (14) et les connexions $\left(\chi_{i j}, \varphi_{i j}\right)$.

Nous allons maintenant montrer qu'il existe un raffinement canonique de la seconde chaîne (14) moyennant la première chaîne (14) et un certain système de connexions $\left(\chi_{j i}^{*}, \varphi_{j i}^{*}\right)$ qui est isomorphe au précédent.

Pour ce but nous énoncerons les définitions suivantes:

$$
\left.\begin{array}{c}
e_{i}^{j}=d_{j}^{i} \\
i=0,1,2, \ldots, r, j=0,1,2, \ldots, s ; \\
b_{j i}^{i-1}=a_{i j}^{j-1} \\
i=1,2, \ldots, r, j=1,2, \ldots, s .
\end{array}\right\}
$$

Pour $i=r$, les $a_{i j}^{j-1}(j=1,2, \ldots, s)$ ont déjà été définis (voir les $\left(25^{\prime \prime}\right)$ ); il en est de même des $d_{j}^{r}$ (voir les $\left(24^{\prime}\right)$ ).

Cela fait, je dis qu'on a pour chaque $j=1,2, \ldots, s$

$$
\left.\begin{array}{c}
b_{j}=e_{0}^{j} \geqq e_{1}^{j} \geqq \ldots \geqq \begin{array}{c}
e_{i-1}^{j} \geqq e_{i}^{j} \geqq \ldots \geqq e_{r-1}^{j} \geqq e_{r}^{j}=\omega_{0}, \\
e_{i}^{j} \leqq e_{i}^{j-1},
\end{array} \\
i=0,1,2, \ldots, r, j=1,2, \ldots, s .
\end{array}\right\}
$$


On a, en effet, $e_{1}^{j}=d_{j}^{1} \leqq b_{j}=e_{0}^{j}$ puis $e_{i-1}^{j}=d_{j}^{i-1}, e_{i}^{j-1}=d_{j-1}^{i} e_{i}^{j}=d_{j}^{i}, d_{j-1}^{i} \geqq$ $\geqq d_{j}^{i}$ et, enfin, $\left(24^{\prime}\right)$; d'où les (29).

Je considère maintenant l'intervalle $b_{j-1} \geqq b_{j}$ et les chaînes

$$
\begin{gathered}
b_{j-1}=e_{0}^{j-1} \geqq e_{1}^{j-1} \geqq \ldots \geqq e_{i-1}^{j-1} \geqq e_{i}^{j-1} \geqq \ldots \geqq e_{r-1}^{j-1} \geqq e_{r}^{j-1}=\omega_{0}, \\
b_{j}=e_{0}^{j} \geqq e_{1}^{j} \geqq \ldots \geqq e_{i-1}^{j} \geqq e_{i}^{j} \geqq \ldots \geqq e_{r-1}^{j} \geqq e_{r}^{j}=\omega_{0} .
\end{gathered}
$$

Le quadrilatère $\left(b_{j i}^{i-1}, e_{i}^{j} ; e_{i-1}^{j}, e_{i}^{j-1}\right)$ est évidemment un sousquadrilatère de $\left(e_{i-1}^{j-1}, \omega_{0} ; e_{i-1}^{j}, e_{i}^{j-1}\right)$; car, en vertu des (27), (28) et de la troisième des (25), on a

$$
e_{i-1}^{j-1} \geqq b_{j i}^{i-1} \geqq e_{i-1}^{j} \geqq e_{i}^{j} \geqq \omega_{0}, \quad e_{i-1}^{j-1} \geqq b_{j i}^{i-1} \geqq e_{i}^{j-1} \geqq e_{i}^{j} \geqq \omega_{0} .
$$

Mais, puisque $\left(b_{j i}^{i-1}, e_{i}^{j}\right.$; $\left.e_{i-1}^{j}, e_{i}^{j-1}\right)$ est identique à $\left(a_{i j}^{j-1}, d_{j}^{i} ; d_{j}^{i-1}, d_{j-1}^{i}\right)$ pour chaque $i=1,2, \ldots, r$ et $j=1,2, \ldots, s$ on aura par les (25)

$$
\begin{aligned}
& b_{j i}^{i-1} / e_{i-1}^{j} \longleftrightarrow e_{i}^{j-1} / e_{i}^{j} \bmod \tilde{\chi}_{i j}, \tilde{\varphi}_{i j}, \\
& b_{j i}^{i-1} / e_{i}^{j-1} \rightleftarrows e_{i-1}^{j} / e_{i}^{j} \bmod \chi_{i j}, \varphi_{i j}, \\
& i=1,2, \ldots, r, \quad j=1,2, \ldots, s .
\end{aligned}
$$

Si donc nous définissons (pour chaque $i=1,2, \ldots, r$ et $j=1,2, \ldots, s$ )

$$
b_{j i}^{0}=b_{j i}=\tilde{\varphi}_{1 j} \tilde{\varphi}_{2 j} \ldots \tilde{\varphi}_{i-1, j} \tilde{\varphi}_{i j}\left(e_{i}^{j-1}\right) ; b_{j 0}=b_{j-1},
$$

nous aurons $b_{j i} \in b_{j-1} / b_{j}$ et $b_{j, i-1} \geqq b_{j i}$. Par conséquent:

$$
\left.\begin{array}{c}
b_{j, i-1} / b_{j i} \leftrightarrow e_{i-1}^{j-1} / b_{j i}^{i-1} \bmod \tilde{\chi}_{i-1, j} \ldots \tilde{\chi}_{2 j} \tilde{\chi}_{1 j}, \tilde{\varphi}_{1 j} \tilde{\varphi}_{2 j} \ldots \tilde{\varphi}_{i-1, j}, \\
i=1,2, \ldots, r, \quad j=1,2, \ldots, s .
\end{array}\right\}
$$

Ici, on a, d'après les relations $(28)$, les troisièmes $\left(25^{\prime \prime}\right)$ et les (27) $b_{s i}^{i-1}=$ $=a_{i s}^{s-1}=d_{s-1}^{i}=e_{i}^{s-1}(i=1,2, \ldots, r)$ et les relations (31) conservent leur sens même pour $j=s$. On a, du reste, par les (28), les secondes (25") et les (27) $b_{j r}^{r-1}=a_{r j}^{j-1}=d_{j}^{r-1}=e_{r-1}^{j}(j=1,2, \ldots, s)$ et je pose (pour des raisons de symétrie par rapport à la quatrième des $\left(25^{\prime \prime}\right), b_{0 r}^{r-1}=e_{r-1}^{0}$.

On en déduit, pour $j=1$

$$
b_{1, i-1} / b_{1 i} \leftrightarrow a_{i-1} / b_{1 i}^{i-1} \bmod \tilde{\chi}_{i-1,1} \ldots \tilde{\chi}_{21} \tilde{\chi}_{11}, \tilde{\varphi}_{11} \tilde{\varphi}_{21} \ldots \tilde{\varphi}_{i-1,1}
$$

Enfin, si l'on prend la chaîne

$$
b_{s i}=e_{i}^{s-1}, \quad i=0,1,2, \ldots, r
$$

pour un raffinement canonique de $b_{s-1} \geqq b_{s}$, on aura (par la seconde relation (30), pour $j=s-1$ ),

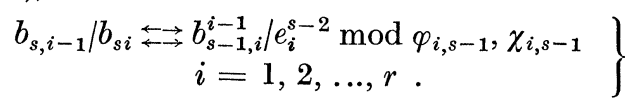

Le théorème de Schreier est maintenant la conséquence des relations $\left(21^{\prime}\right)$, $\left(31^{\prime}\right),\left(25^{\prime \prime}\right),(31),\left(26^{\prime}\right),(25),\left(30^{\prime}\right),(30)$ et des définitions (27), (28).

Je dirai que les raffinements $a_{i j}, b_{j i}$ que nous avons ainsi obtenus forment un système de raffinements mutuels canoniques des chaînes (14).

Les quotients $a_{i, j-1} / a_{i j}$ et $b_{j, i-1} / b_{j i}$ sont les quotients conjugués de ce système de raffinements; ces quotients sont, d'après le théorème, toujours isomorphes 
au quotient intermédiaire $d_{j-1}^{i-1} / a_{i j}^{j-1}$; en particulier, chacun des quotients, $\Omega / a_{11}$ et $a_{r, s-1} / \omega_{0}=b_{s, r-1} / \omega_{0}$ est isomorphe à soi-même. Ce système de raffinements, on le voit, dépend en général du choix des quadrilatères $\left(a_{i j}^{j-1}, d_{j}^{i}\right.$; $\left.d_{j}^{i-1}, d_{j-1}^{i}\right)$ et des connexions parfaites $\left(\chi_{i j}, \varphi_{i j}\right)$ et $\left(\tilde{\chi}_{i j}, \tilde{\varphi}_{i j}\right)$.

Corollaire. Supposons que $P$ soit un espace $\Delta$ modulaire; dans ce cas le théorème de Schreier est vrai pour chaque paire de chaînes aux extrémités communes.

3.2. Les raffinements canoniques, généralités. Il n'est pas difficile de se rendre compte de ce que la seconde des deux démonstrations que nous avons données du théorème de Schreier, n'est, au fond, que la description d'une méthode permettant de faire correspondre, dans tout espace $\Delta$ (2.1) (qu'il satisfasse ou non à la supposition $H$ ) à chaque paire de chaînes aux extrémités communes, au moins un système de raffinements mutuels canoniques; mais, si l'espace $\Delta$ en question n'est pas modulaire (ou s'il ne satisfait pas à la supposition $\boldsymbol{H}$ ) il ne peut, évidemment, y être question, en général, de raffinements (mutuels canoniques) isomorphes.

Dans le cas des structures, les (sous) - quadrilatères de la forme $(a \vee b$, $a \wedge b ; a, b)$ et les connexions de Dedekind qui leur correspondent, conduisent, comme il est facile de s'en convaincre, au système de raffinements de Zassenhaus; on a, en effet, dans chaque structure $d_{j}^{i}=a_{i} \wedge b_{j}(i=0,1,2, \ldots, r$; $\left.j=0,1,2, \ldots, s), a_{i j}^{j-1}=d_{j}^{i-1} \vee d_{j-1}^{\prime} 1=1,2, \ldots, r ; j=1,2, \ldots, s\right)$. D'après cela (et puisque les $\left(25^{\prime}\right)$ ont un sens même si les $\chi_{i j}, \varphi_{i j}$ ne sont pas des isomorphismes), on aura $\left(\ldots\left(\left(\left(a_{i j}^{j-1} \vee d_{j-2}^{i}\right) \vee d_{j-3}^{i}\right) \vee d_{j-4}^{i}\right) \vee \ldots\right) \vee d_{0}^{i}=a_{i} \vee$ $\vee\left(a_{i-1} \wedge b_{j}\right)$, etc. (voir les $\left(25^{\prime}\right)$ !). On sait du reste que, pour que les raffinements de Zassenhaus soient semblables d'en bas pour chaque paire de chaînes (14) il faut et il suffit que la structure soit modulaire; en ce cas ces raffinements sont même isomorphes.

Je n'insisterai pas davantage ici sur les détails de la construction des raffinements mutuels canoniques; ces détails, ils me paraissent suffisamment éclaircis par ce qui précède.

En ce qui concerne l'emploi des connexions multivoques dans la théorie des raffinements, il en sera question dans la seconde partie de ce mémoire.

Nous allons maintenant appliquer les principes précédents aux cas des structures et des semigroupes (théorie de la divisibilité).

\section{§ 4. LES STRUCTURES}

4.1. Définition. J'appelle $W$-structure, toute structure qui est un espace $\Delta$ modulaire. Ceci équivaut à dire, on s'en convainc facilement, que quels que soient les éléments $a, b \in S$ la relation d'isomorphisme (de structure!)

$$
a \vee b / a \leftrightarrows b / a \wedge b \bmod \chi, \varphi
$$

est vérifiée. 
Toute structure modulaire (c'est-à-dire satisfaisant à l'axiome de Dedekind) est, naturellement, une $W$-structure mais, la réciproque n'est pas vraie, ainsi que l'a montré M. WARD [18] qui a, d'ailleurs, été le premier à considérer les $W$-structures.

La proposition suivante est une conséquence immédiate du $\S 3$.

4.2. Théorème. Deux chaînes aux extrémités communes possèdent, dans toute $W$-structure, un système de raffinements mutuels canoniques; ces raffinements sont des chaînes de même longueur dont les quotients conjugués sont aux structures isomorphes.

Les raffinements dont il s'agit dans cet énoncé, sont, en général, différents de ceux de Zassenhaus et cela précisément parceque les connexions (33) ne sont, en général, pas même radiales; mais l'intérêt du théorème lui-même vient, à mon avis, de ce que la structure peut ne pas y être modulaire.

4.3. Sur la caractérisation des structures modulaires. C'est M. Ward qui a posé (l. c.) le problème de la caractérisation des structures modulaires en tant que $W$-structures; il a démontré, comme on sait, la proposition suivante:

Pour qu'une $W$-structure soit une structure modulaire il suffit qu'elle soit archimédienne ${ }_{1}$ (ou bien archimédienne ${ }_{2}$ ).

Je vais dans ce qui suit donner, tout en la généralisant un peu, une nouvelle démonstration fort simple de cette proposition; l'intérêt de cette démonstration vient de ce qu'elle s'applique aussi dans la théorie des multistructures (voir la seconde partie de ce mémoire), tandis que le raisonnement de M. Ward lui-même est plutôt ,,verbandtheoretisch".

4.31. Définition. Je dis qu'un ensemble partiellement ordonné $P$ est méromorphe lorsqu'il satisfait à la condition suivante:

$\mathbf{Q}^{\prime}$. Soient $a, b, c \in P$ tels que $a \geqq b \geqq c$ et tels qu'on ait l'isomorphisme $a / c \rightleftarrows$ $\rightleftarrows b / c$; alors on $a \quad a=b$.

De même, je dis que $P$ est holomorphe lorsqu'il satisfait la condition suivante:

$\mathbf{Q}^{\prime \prime}$. Soient $a, b, c \in P$ tels que $a \geqq b \geqq c$ et tels qu'on ait l'isomorphisme $a / b \leftrightarrows a / c$; alors, on $a b=c$.

4.32. Lemme. Un ensemble partiellement ordonné archimédien (archimédien $_{2}$ ) est toujours méromorphe (holomorphe).

La démonstration est analogue à celle qu'on donne habituellement dans la théorie des groupes pour prouver qu'un groupe à structure groupale archimédienne $_{1}\left(\right.$ archimédienne $_{2}$ ) n'a pas de méromorphismes (holomorphismes) véritables.

4.33. Théorème ( $M$. Ward). Soit S une $W$-structure méromorphe (holomorphe); alors, $S$ est modulaire.

Démonstration. Soient $a, b \in S$ et $p^{\prime} \in b / a \wedge b$, c'est-à-dire $b \geqq p^{\prime} \geqq a \wedge$ $\wedge b$. On a évidemment 


$$
\begin{aligned}
& \left.\begin{array}{l}
a \vee b \geqq a \vee p^{\prime} \geqq a, \\
b \geqq b \wedge\left(a \vee p^{\prime}\right) \geqq p^{\prime} \geqq a \wedge b,
\end{array}\right\} \\
& \left.\begin{array}{l}
a \vee\left[b \wedge\left(a \vee p^{\prime}\right)\right]=a \vee p^{\prime}, \\
a \wedge\left[b \wedge\left(a \vee p^{\prime}\right)\right]=a \wedge p^{\prime}=a \wedge b,
\end{array}\right\}
\end{aligned}
$$

(On a, par exemple, pour la dernière des relations ci-dessus: $b \geqq p^{\prime} \geqq a \wedge b$ donc $a \wedge b \geqq a \wedge p^{\prime} \geqq a \wedge b$ c'est-à-dire $a \wedge p^{\prime}=a \wedge b$; or, $b \geqq b \wedge(a \vee$ $\left.\vee p^{\prime}\right) \geqq a \wedge b$, etc).

Par conséquent, $S$ étant une $W$-structure, on aura les isomorphismes:

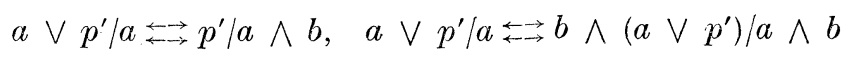

dont la combinaison nous donne l'isomorphisme:

$$
p^{\prime} / a \wedge b \overleftrightarrow{\leftrightarrows} b \wedge\left(a \vee p^{\prime}\right) / a \wedge b
$$

qui entraîne, puisque $S$ est méromorphe, que $b \wedge\left(a \vee p^{\prime}\right)=p^{\prime}$. Or, ceci prouve, d'après un théorème de Ore [5], que $S$ est modulaire. Pour le cas d'une structure $S$ holomorphe on peut appliquer la dualité. Q. E. D.

Le théorème origin aire de M. Ward est maintenant la conséquence immédiate du lemme 4.32 et du théorème 4.33 .

Remarque. On s'aperçoit aisément que dans la démonstration du théorème 4.33 ce n'est qu'une partie seulement de la condition $Q^{\prime}$ qui est intervenue; c'est que l'élement $b \wedge\left(a \vee p^{\prime}\right)$ n'est jamais arbitraire mais il est, au contraire, la ,fermeture“" de $p^{\prime}$ dans $b / a \wedge b$. On est ainsi conduit à envisager les structures $S$ satisfaisantes à la condition suivante:

$Q^{*}$. Soient $a, b \in S$ et soit $p^{\prime} \in b / a \wedge b$ tel qu'on ait l'isomorphisme

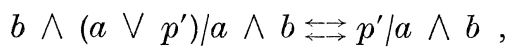

alors, on a $b \wedge\left(a \vee p^{\prime}\right)=p^{\prime}$. Une condition $\mathbf{Q}^{* *}$ s'en déduit par la dualité.

$\mathrm{Or}$, on voit tout de suite que si dans l'énoncé du théorème 4.33 on remplace la condition $\mathbf{Q}^{\prime}$ par la condition moins exigeante $\mathbf{Q}^{*}$, le théorème reste vrai.

\section{$\S$ 5. LES SEMIGROUPES}

Il s'agit dans ce paragraphe de questions se rattachant aux fondements de la théorie de la divisibilité. Cette dernière est d'habitude subordonnée à la théorie d.s (semi)groupes réticulés [19]. Parmi les propositions les plus élémentaires (mais nullement triviales) de ces théories, il y en a, cependant, dont la validité et la signification sont indépendantes de ce que le groupe partiellement ordonné fondamental soit réticulé; ce sont ces propositions-là que nous allons. considérer dans les alinéas suivants, afin d'en tirer quelques conséquences à la lumière du $\S 3$.

5.1. Définitions. Soit $S$ un semigroupe arbitraire (c'est-à-dire un ensemble non vide muni d'une loi de composition $(\cdot)$, qu'on appelle multiplication, et qui est unique et associative). 
Je dis que $S$ est à élément unité à gauche (à droite), s'il existe un $1^{\prime} \in S\left(1^{\prime \prime} \in S\right)$ tel que pour tout $a \epsilon S$ on ait $1^{\prime} . a=a\left(a .1^{\prime \prime}=a\right)$; et je dis que $S$ est à élément unité lorsqu'il l'est simultanément à droite et à gauche. De même, je dis que $S$ est à élément zéro, s'il existe un $0 \in S$ tel qu'on ait 0 . $a=a .0=0$ pour chaque $a \in S$.

Soient $a, b, c \in S$ tels que $a=b c$; alors, je dis que $b$ est un diviseur $\dot{a}$ gauche de $a$, tandis que $c$ en est un diviseur à droite. Symboles: $a \varrho^{\prime} b, a \varrho^{\prime \prime} c$. Les relations binaires $\varrho^{\prime}, \varrho^{\prime \prime}$ seront dites les divisibilités à gauche et à droite (dans $S$ ).

Je dis que $S$ est simplifiable à gauche (à droite) lorsque l'égalité $a x=a y$ $(x a=y a)$ entraîne toujours l'égalité $x=y$; et je dis que $S$ est simplifiable lorsqu'il l'est à la fois à gauche et à droite.

Enfin, j'appelle semigroupe à division ou semigroupe divisionnaire tout semigroupe $S$ tel que 1. $S$ soit à élément unité et à élément zéro, $2 . S-(0)$ soit sinıplifiable, 3 . on ait $a S=S a$ pour chaque $a \in S$, 4. le sousgroupe $U$ des unités de $S$ se réduise à l'identité.

On a d'abord la proposition bien connue suivante:

5.2. Lemme. La relation $\varrho^{\prime}$ est une relation de quasi-ordre, c'est-à-dire reflexive. et transitive, dans $S$, pourvu que $S$ soit à élément unité à droite. ${ }^{9}$ )

5.3. Les ensembles $S^{\prime}, S^{\prime \prime}$. Soit $S$ un semigroupe à élément unité à droite; alors, la divisibilité $\varrho^{\prime}$, y est, d'après 5.2, une relation de quasi-ordre [19]. Or, on sait qu'en ce cas, la relation $a \mathrm{P}^{\prime} b(a, b \in S)$ définie par:

$\mathrm{P}^{\prime}$. On a a $\mathrm{P}^{\prime} b$ toutes les fois qu'on $a$ a $\varrho^{\prime} b$ et $b \varrho^{\prime} a$,

est une relation d'équivalence dans $S$. C'est l'ensemble quotient $S / P^{\prime} q u$ 'elle définit que nous désignerons dans la suite par $S^{\prime}=S / P^{\prime}$; on sait [19] que l'ensemble $S^{\prime}$ est partiellement ordonné par une relation $\leqq$ telle, qu'on ait $K_{x} \leqq K_{y}$ si et seulement si que la relation $x^{*} \varrho^{\prime} y^{*}\left(x^{*} \in K_{x}\right.$ et $\left.y^{*} \in K_{y} ; K_{x}, K_{y} \in S^{\prime}\right)$ est vérifiée ou, ce que revient au même, que $y$ soit un diviseur à gauche, de $x$; au lieu de $K_{x} \leqq K_{y}$ j'écrirai aussi $K_{y} \geqq K_{x}$ [19].

On aura pareillement, $S$ étant à élément unité à gauche, une relation d'équivalence $a P^{\prime \prime} b(a, b \in S)$ définie par $\left\langle\left\langle a \varrho^{\prime \prime} b\right.\right.$ et $\left.\left.b \varrho^{\prime \prime} a\right\rangle\right\rangle$ et un ensemble quotient $S^{\prime \prime}=S / P^{\prime \prime}$.

Lorsque, dans la suite, nous parlerons de l'ordre partiel de l'ensemble $S^{\prime}$ il s'agira toujours de celui défini par la relation $\leqq$ ci-dessus.

5.4. Lemme. Soit $S$ un semigroupe à élément unité 1 et soit $U$ le groupe des unités de $S$; alors toute classe d'équivalence de $S$ suivant $\mathrm{P}^{\prime}$ c'est-à-dire tout élément $K_{x} \epsilon S^{\prime}$ est une somme de classes résiduaires à gauche de $S$ par rapport à $U$. (La démonstration fait usage de l'axiome de choix).

$\left.{ }^{9}\right)$ Il en est de même de $\varrho^{\prime \prime}$, pourvu que $S$ soit à élément unité à gauche. 
5.5. Lemme. Pour que $\varrho^{\prime \prime} \rightarrow \varrho^{\prime} c^{\prime} e s t$-à-dire, pour que la relation a $\varrho^{\prime \prime} b$ entraîne $a \varrho^{\prime} b$ il faut et il suffit que pour chaque élément $a \in S$ on ait $S a \subseteq a S .{ }^{10}$ )

Il en résulte que $a \varrho^{\prime} b$ entraîne toujours $a c \varrho^{\prime} b c$ pour chaque $c \in S$.

La vérification des lemmes 5.4 et 5.5 n'offre aucune difficulté.

5.6. Lemme. Soit $S$ un semigroupe à élément unité à droite et tel que $\varrho^{\prime \prime} \rightarrow \varrho^{\prime}$; alors, l'ensemble $S^{\prime}$ est un semigroupe par rapport à la multiplication ordinaire des classes $K_{x} \in S^{\prime}$. Ce semigroupe $S^{\prime}$ possède les propriétés suivantes: $1^{\circ}$ il est à él'ément unité à droite, $2^{\circ}$ la divisibilité à gauche y coincide (en tant que relation de quasi-ordre) avec l'ordre partiel de $S^{\prime}$ (5.3, dernière remarque), $3^{\circ}$ la divisibilité à droite y entrâ̂ne la divisibilité à gauche (c'est-à-dire, on $a \varrho^{\prime \prime} \rightarrow \varrho^{\prime}$ dans $\left.S^{\prime} !\right), 4^{\circ}$ l'ordre partiel de $S^{\prime}$ est homogène par rapport $\dot{a}$ la multiplication des classes, c'est-à-dire que $K_{a} \leqq K_{b}$ entraîne $K_{c} K_{a} \leqq K_{c} K_{b}$ et $K_{a} K_{c} \leqq K_{b} K_{c}$ pour chaque $K_{c} \in S^{\prime}$.

Démonstration. On a par définition $S^{\prime}=S / \mathrm{P}^{\prime}, \mathrm{P}^{\prime}$ étant la relation d'équivalence définie à 5.3. Or, il est clair que $a \mathrm{P}^{\prime} a^{*}\left(a, a^{*} \in S\right)$ entraîne $c a \mathrm{P}^{\prime} c a^{*}$ pour chaque $c \in S$; et je dis qu'on a également $a c \mathrm{P}^{\prime} a^{*} c$ pour chaque $c \in S$. On a, en effet $a=a^{*} d, a^{*}=a d^{\prime}$ et par suite aussi $a c=a^{*} d c, a^{*} c=a d^{\prime} c$ ce qui, en vertu de la supposition $\varrho^{\prime \prime} \rightarrow \varrho^{\prime}$, entraîne (5.5), $a c=a^{*} c d_{1}, a^{*} c=a c d_{1}^{\prime}$ c'est-à-dire précisément $a c \mathrm{P}^{\prime} a^{*} c$. Ainsi l'ensemble $S^{\prime}$ est un système multiplicatif par rapport à la multiplication ordinaire des classes: $K_{a} K_{b}=K_{a b}$; $K_{a}, K_{b} \in S^{\prime}$; on a donc l'homomorphisme $S \sim S^{\prime}$ qui entraîne en particulier que $S^{\prime}$ est un semigroupe.

Les propriétés $1^{\circ}-4^{\circ}$ du semigroupe $S^{\prime}$ sont essentiellement des conséquences de l'homomorphisme $S \sim S^{\prime}$; montrons-le pour les propriétés $2^{\circ}, 3^{\circ}$.

Soient d'abord $K_{a}, K_{b}, K_{c} \in S^{\prime}$ telles que $K_{a}=K_{b} K_{c}$. Je dis qu'on a $K_{a} \leqq K_{b}$; on a en effet $K_{a}=K_{b c}$ donc $a \mathrm{P}^{\prime} b c$ c'est-à-dire, en particulier, $a=b . c d$ ce qui prouve que $a \varrho^{\prime} b$ et, partant, $K_{a} \leqq K_{b}$ (5.3).

Réciproquement on tire de $K_{a} \leqq K_{b}$ que $a \varrho^{\prime} b$, donc $a=b c$ et par suite, vu que $S \sim S^{\prime}, K_{a}=K_{b} K_{c}$, ce qui achève la vérification de $2^{\circ}$.

Supposons maintenant qu'on ait $K_{a}=K_{d} K_{b}$; il faut montrer qu'il en résulte $K_{a}=K_{b} K_{c}$. Or, on a $K_{a}=K_{a b}$, donc $a \mathrm{P}^{\prime} d b$, c'est-à-dire que $a=d b f$; on a, d'autre part, $\varrho^{\prime \prime} \rightarrow \varrho^{\prime}$ et par conséquent $d b=b d^{\prime}$, donc $a=b . d^{\prime} f=b c$ avec $c=d^{\prime} f$. Il en résulte enfin $K_{a}=K_{b} K_{c}$ : c'est la propriété $3^{\circ}$ de l'énoncé.

5.\%. Lemme. Soit $S$ un semigroupe à élément unité 1 , simplifiable à gauche et tel que $\varrho^{\prime \prime} \rightarrow \varrho^{\prime}$; alors le semigroupe $S^{\prime}$ jouit des mêmes propriétés et coïncide avec l'ensemble des classes résiduaires à gauche de $S$ suivant le sousgroupe $U$ des unités de $S$.

Démonstration. Soit $K_{a} \in S^{\prime}$ et $a^{*} \in K_{a}$, donc $a=a^{*} d, a^{*}=a f$; on en tire $a=a f d, a^{*}=a^{*} d f$ et comme $S$ est simplifiable à gauche, il en résulte $1=d f$,

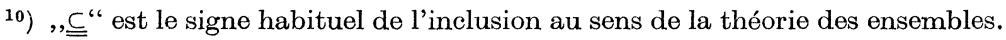


$1=f d$, ce qui prouve que $d, f \epsilon U$, donc $K \subseteq a U$. Or, on a aussi (5.4) $a U \subseteq K$, donc $K=a U$. Le reste de la démonstration s'achève comme à 5.6.

Corollaire. 1. On a $U a \subseteq a U$ pour chaque $a \in S$. 2. La relation $K_{c} K_{a} \leqq$ $\leqq K_{c} K_{b}$ entraîne $K_{a} \leqq K_{b}$.

Dorénavant nous supposerons que $S$ est un semigroupe divisionnaire (5.1); alors, $a \varrho^{\prime} b\left(\leftrightarrow a \varrho^{\prime \prime} b\right)$ signifie (5.3) $a \leqq b$ et l'on a $0 \leqq a \leqq 1$ pour chaque $a \in S$.

5.8. Lemme. Soit $S$ un semigroupe divisionnaire; alors, tout quadrilatère $(M, d ; a, b), d>0$, tel que $b=v M, d=v a$, est un quadrilatère tel que $a=M \tilde{v}$, $d=b \tilde{v}$ et tel qu'on ait les isomorphismes (au sens de l'ordre partiel)

$$
M / a \rightleftarrows b / d, \quad M / b \rightleftarrows a / d .
$$

Démonstration. D'abord, puisque $U=1$, il suit que $S$ est partiellement ordonné par la divisibilité. Cela étant, je considère $p \in M / a$, c'est-à-dire $M \geqq$ $\geqq p \geqq a$ et je définis

$$
\chi(p)=v p, \quad p \in M / a .
$$

Il est clair que $\chi(p) \epsilon b / d$, c'est-à-dire que $b \geqq \chi(p) \geqq d$. Soit maintenant $p^{\prime} \in b / d$. Puisque $b=v M$, c'est-à-dire $v \geqq b$, on aura a fortiori $v \geqq p^{\prime}$, ce qui équivaut à $p^{\prime}=v z$ pour un certain $z \in S$. De $b \geqq p^{\prime} \geqq d$ il résulte, d'ailleurs, $v M \geqq v z \geqq v a$ et, par suite, $M \geqq z \geqq a$. Je définis alors

$$
\varphi\left(p^{\prime}\right)=z \quad\left(p^{\prime}=v \varphi\left(p^{\prime}\right)\right), \quad p^{\prime} \epsilon b / d
$$

et je dis qu'on a $M / a \overleftrightarrow{\longleftrightarrow} b / d \bmod \chi, \varphi$.

Ces fonctions $\chi, \varphi$ sont en effet monotones; puis, on a $v \varphi \chi(p)=\chi(p)=v p$, donc $\varphi \chi(p)=p$ et $\chi \varphi\left(p^{\prime}\right)=v \varphi\left(p^{\prime}\right)=p^{\prime}$.

Maintenant, puisque $M \geqq a$ et $b \geqq d$, on aura $a=M v_{1}, d=b v_{2} \operatorname{donc} d=v$. . $M v_{1}=v M . v_{1}=b v_{1}$. Donc $v_{1}=v_{2}=\tilde{v}$. Si je pose

$$
\tilde{\chi}(q)=q \tilde{v}, \quad q \in M / b ; \quad \tilde{\varphi}\left(q^{\prime}\right) \tilde{v}=q^{\prime}, \quad q^{\prime} \epsilon a / d,
$$

j'obtiendrai d'une façon analogue $M / b \rightleftarrows a / d \bmod \tilde{\chi}, \tilde{\varphi}$ et le lemme est démontré.

5.81. Lemme. Soit $S$ un semigroupe divisionnaire, alors, pour qu'un quadrilatère $(1, \omega ; a, b), \omega>0$, possède un sousquadrilatère $(M, d ; a, b)$ tel que $b=$ $=v M, d=v a$ il faut et il suffit qu'il $y$ ait un $d \in S$ tel que $d \leqq a, d \leqq b, d \geqq \omega$, $d \geqq b a$.

5.82. Corollaire. $S$ étant un semigroupe divisionnaire, pour qu'un quadrilatère $(\Omega, \omega ; a, b), \omega>0$, possède un sousquadrilatère $(M, d ; a, b)$ tel que $b=v M$, $d=v a$, il faut et il suffit qu'il $y$ ait un $d \in S$ tel que $d \leqq a, d \leqq b, d \geqq \omega, d \geqq$ $\geqq b a^{\prime}$ avec $a=\Omega a^{\prime}$.

5.83. Corollaire. Tout semigroupe divisionnaire satisfait à la supposition $\boldsymbol{H}$ (avec $\omega_{0}=0$ !). 
5.9. Théorème. Dans tout semigroupe divisionnaire $S$, deux chaînes aux extrémités communes $\Omega>0(\Omega \leqq 1)$ possèdent toujours au moins un système de raffinements isomorphes.

Chacune des propositions 5.81-5.9 est une conséquence simple de la proposition précédente (ainsi que, quant à 5.9 , du $\S 3$ ).

5.91. Remarque. La question reste ouverte de savoir sous quelles conditions le théorème 5.9 conserve-t-il sa validité lorsqu'on y remplace 0 par un $\omega_{0} \epsilon S$ tel que $\omega_{0} \neq 0$, donc tel que $\omega_{0}>0$ (et tel que $\omega_{0}<\Omega$ )? Il s'agit, bien entendu, de conditions autres (s'il en existe) que celles équivalant à ce que le semigroupe divisionnaire $S$ soit une structure par rapport à l'ordre partiel de $S$ (5.3).

Cette question n'est pas aisée; il y faut, à mon avis, remplacer (dans la supposition $\boldsymbol{H}$ ) les isomorphismes (34) et $(\tilde{34})$ de 5.8 par des correspondances (isomorphes) distinctes des correspondances $(\chi, \varphi),(\tilde{\chi}, \tilde{\varphi})$ de 5.8. Or, dans le cas où le semigroupe divisionnaire $S$ est une structure (archimédienne ${ }_{2}$ ), ces dernières correspondances coïncident avec les connexions de Dedekind de n'importe quel quadrilatère $(a \vee b, a \wedge b ; a, b)$ [19], Ch. XIV, §§ 4 et 13, il n'en est plus de même dans le cas plus général où le semigroupe $S$ est

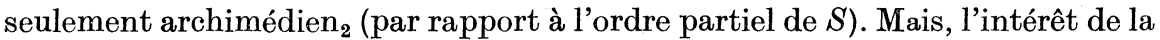
question vient surtout de ses étroits liens avec le problème de la divisibilité des entiers algébriques (voir plus loin).

5.92. Et voici, pour terminer, une application de ce qui précède aux anneaux d'intégrité ayant un élément unité (par anneau d'intégrité j'entends un anneau qui n'a pas de diviseurs de zéro; si, de plus, il est commutatif, c'est un domaine d'intégrité).

Considérons l'ensemble $\Pi$ (partiellement ordonné par l'inclusion ordinaire C) de tous les idéaux bilatéralement principaux d'un anneau d'intégrité $R$ qui est à élément unité; cet ensemble $\Pi$ jouit des propriétés principales suivantes (qu'on démontre comme dans le cas où tous les idéaux de $R$ seraient principaux $\left.^{11}\right)$ :

$1^{\circ}$ Pour chaque $\alpha \epsilon \Pi$ on a $\alpha=(a)$ où $a \epsilon R$ est tel que $a R=R a, 2^{\circ}$ l'ensemble $\Pi$ est un semigroupe par rapport à la multiplication ordinaire des idéaux, $3^{\circ}$ soient $\alpha, \beta \in \Pi$ tels que $\alpha \subseteq \beta$; alors il existe des idéaux $\gamma, \gamma^{\prime} \in \Pi$ tels que $\alpha=\beta \gamma=\gamma^{\prime} \beta, 4^{\circ}$ soient $\alpha, \xi, \eta \in \Pi, \alpha \supset(0)$ tels que $\alpha \xi=\alpha \eta(\xi \alpha=\eta \alpha)$; alors $\xi=\eta, 5^{\circ}$ soient $\alpha, \beta \epsilon \Pi, \alpha \supset(0), \beta \supset(0)$; on a alors entre les paires d'anneaux résiduaires $R / \alpha, \beta / \alpha \beta$ et $R / \beta, \alpha / \alpha \beta$, les isomorphismes de module suivants (permis par $R$ à gauche resp. à droite) $R / \alpha \cong \beta / \alpha \beta, R / \beta \cong \alpha / \alpha \beta$; il en résulte pour $\alpha, \beta, \gamma \in \Pi$ tels que $(0) \subset \alpha \subseteq \beta$, les isomorphismes de module $\beta \gamma / \alpha \gamma \cong$ $\cong \beta / \alpha \cong \gamma \beta / \gamma \alpha$ et les isomorphismes au sens de l'ordre partiel $\beta \gamma / \alpha \gamma \leftrightarrows \beta / \alpha \leftrightarrows$

$\left.{ }^{11}\right)$ Voir, par exemple, N. Jacobson, Theory of Rings, Chapter III. 
$\rightleftarrows \gamma \beta / \gamma \alpha$ (dans ces derniers, $\xi / \eta=$ l'ensemble de tous les $\Theta \epsilon \Pi$ tels que $\xi \supseteqq$ $\supseteqq \Theta \supseteqq \eta$.

L'ensemble $\Pi$ est, d'après ces propriétés, un semigroupe divisionnaire et le théorème 5.9 y est, par conséquent, satisfait pour chaque paire de chaînes aux extrémités communes $(a) \supset(0)$ où $a \epsilon R$ est tel que $a R=R a$.

Reste à montrer que l'ensemble $\Pi$ n'est pas, en général, une structure (par rapport à l'ordre partiel $\subseteq \mathrm{de} \Pi$ ). On a d'abord à ce sujet la proposition suivante:

5.93. Théorème. Soit $R$ un domaine d'intégrité ayant un élément unité; alors, pour que l'enseble $\Pi$ de tous les idéaux principaux de $R$ soit une structure archimédienne (1.2), il faut et il suffit que $R$ satisfasse au ,Satz von der eindeutigen Primfaktorzerlegung $\left.{ }^{12}\right)$; en ce cas, la structure $\Pi$ est distributive.

Démonstration. La condition est évidemment suffisante. Quant à la nécessité, voir [19], pages 217-8, et 235-6 (mais auparavant, la dualité doit y être appliquée aux résultats des pages $235-6$ ).

5.94. Maintenant, on sait que l'ordre principal d'un corps de nombres algébriques ne satisfait pas ordinairement au ,,Satz von der eindeutigen Primfaktorzerlegung “; ainsi l'ensemble ( $\operatorname{archimédien~}_{2}$ ) $\Pi$ de tous les idéaux principaux d'un tel ordre ne saurait, en général, être une structure.

\section{LITERATURE}

[1] O. Schreier, Über den Jordan-Hölderschen Satz, Abhandl. Hamburg, vol. 6 (1930), $300-302$.

[2] H. Zassenhaus, Zum Satz von Jordan-Hölder-Schreier, Abhandl. Hamburg, vol. 10 (1934), $106-108$.

[3] A. Kurosch, Eine Verallgemeinerung des Jordan-Hölderschen Satzes, Math. Annalen, vol. 111 (1935), 13-18.

[4] A. Kurosch, Le théorème Jordan-Hölder dans les structures arbitraires, Recueil de travaux dédiés à la mémoire de l'Acad. D. A. Gravé, Moscou, 1940, 110-116 (en russe).

[5] O. Ore, On the foundations of abstract algebra, I. Annals of Math., vol. 36 (1935), $406-437$.

[6] O. Ore, On the theorem of Jordan-Hölder, Transact. of Amer. Math. Soc., vol. 41 (1937), 266-275.

[7] A. Halanay, Le théorème Jordan-Hölder dans la théorie des structures, Disquisit. Math. et Phys. t. VII, fasc. $1-4$ (1948), 3-23.

[8] A. I. Ouzkov, On the Jordan-Hölder theorem, Recueil Math. Moscou t. 4 (nouvelle série) 1938, 31-43 (en russe, avec un résumé en anglais).

[9] E. George, Über den Satz von Jordan-Hölder-Schreier, Journal für reine u. angewandte Math. vol. 180 (1939), 110-120.

[10] V. Koŕinek, Der Schreiersche Satz und das Zassenhaussche Verfahren in Verbänden, Věstník Král. České Spol. Nauk, vol. XIV (1941), 1-29.

[11] O. Ore, Chains in partially ordered sets, Bull. of Amer. Math. Soc. vol. 49 (1943), $558-566$.

12) C'est-à-dire que chaque élément $a \epsilon R$ (à part le zéro et les unités) soit représentable d'une façon unique comme produit d'éléments irréductibles (abstraction faite de l'ordre des facteurs et des unités s'y présentant). 
[12] P. Lorenzen, Eine Bemerkung zum Schreierschen Verfeinerungssatz, Math. Zeitschrift, vol. 49 (1945), $647-653$.

[13] A. H. Lipschitz, Du théorème Jordan-Hölder dans les structures, Recueil Math. Moscou t. 24 (nouvelle série), 1949, 227-235 (en russe).

[14] D. Barbilian, Normalités involutives, locales respectivement globales (en roumain, avec des résumés en français et en russe). Etudes et recherches mathématiques, vol. IV, 1953.

[15] M. Benado, La notion de normalité et les théorèmes de décomposition de l'Algèbre, Etudes et Recherches Math. vol. I, fasc. 2, 1950, pp. 282-305 (en roumain, avec des résumés en français et en russe).

[16] M. Benado, Sur les théorèmes de décomposition de l'Algèbre, Etudes et Recherches Math. vol. III , fasc. $1-2,1952$, pp. 263-288 (en roumain, avec des résumés en français et en russe).

[17] M. Benado, Théorie abstraite des relation de normalité (en roumain avec des résumés en français et en russe). Etudes et recherches mathématiques, vol. IV, 1953, pp. $69-112$.

[18] M. Ward, A characterization of Dedekind structures, Bull. of. Amer. Math. Soc. vol. 45 (1939) pp. 448-451.

[19] G. Birkhoff, Lattice Theory, Amer. Math. Soc. Colloq. Publications, revised edition, New-York, 1948.

\title{
Резюме.
}

\section{ЧАСТИЧНО УПОРЯДОЧЕННЫЕ МНОЖЕСТВА И ТЕОРЕМА ШРЕЙЕРА ОБ УПЛОТНЕНИИ, I}

\author{
МИХАИЛ БЭНАДО (Michail Benado), Бухарест.
}

(Поступило в редакцию 6/II 1953 г.)

Предметом указанной работы является расширение теоремы Шрейера [1] для случая частично упорядоченных множеств, которые не являются структурами, а именно при помощи следующего предположения:

Н. Мы говорим, что частично упорядоченное множество $P$ удовліетворяет предположению $\mathbf{H}$, если существует элемент $\omega_{0}$ так, что для всякого четырехугольника $\left(\Omega, \omega_{0} ; a, b\right)$ можно найти подчетырехугольник $(M, d ; a, b)$ так, что согласно закону частичного упорядочения имеют место изоморфизмы $\eta, \tilde{\eta}[19]$

$$
M / a \rightleftarrows b / d(\eta), \quad M / b \rightleftarrows a / d(\tilde{\eta}) .
$$

Под четырехугольником мы здесь понимаем какие-либо четыре элемента для которых выполняется

$$
\Omega \geqq a \geqq \omega, \quad \Omega \geqq b \geqq \omega .
$$

Подчетырехугольник четырехугольника (1) является всякий четырехугольник вида $(M, d ; a, b)$, для которого

$$
\Omega \geqq M, \quad d \geqq \omega .
$$

Очевидно, что предположение Н является некоторой аналогией ,второй основной теоремы об изоморфизме“". 
Теперь теорему Шрейера об уплотнении можно сформулировать следующим способом:

Две цепи с общими концами $\Omega \geqq \omega_{0}$ из $P$ можно уплотнить по крайней мере одним способом так, что уплотненные цепи изоморфны.

Для доказательства достаточно повторить слово в слово оригинальное доказательство Шрейера посредством двойной индукции. Работа содержит еще одно доказательство этой теоремы, которое более конструктивно.

Далее в работе приведены некоторые применения этой теоремы IШрейера к теории структур и к теории делимости. Но прежде всего эта теорема будет использована во второй части работы для изучения одного довольно большего класса частично упорядоченных множеств, который содержит структуры как частный случай. 